\title{
Some phenomenological aspects of the 3-3-1 model with the Cárcamo-Kovalenko-Schmidt mechanism
}

\author{
H. N. Long, ${ }^{1,2, *}$ N. V. Hop, ${ }^{3, \dagger}$ L. T. Hue, ${ }^{4,5, *}$ N. H. Thao, ${ }^{6, \S}$ and A. E. Cárcamo Hernández ${ }^{7, \|}$ \\ ${ }^{1}$ Theoretical Particle Physics and Cosmology Research Group, Advanced Institute of Materials Science, \\ Ton Duc Thang University, Ho Chi Minh City 700000, Vietnam \\ ${ }^{2}$ Faculty of Applied Sciences, Ton Duc Thang University, Ho Chi Minh City 700000, Vietnam \\ ${ }^{3}$ Department of Physics, Can Tho University, 3/2 Street, Can Tho 900000, Vietnam \\ ${ }^{4}$ Institute for Research and Development, Duy Tan University, Da Nang City 550000, Vietnam \\ ${ }^{5}$ Institute of Physics, Vietnam Academy of Science and Technology, 10 Dao Tan, Ba Dinh, \\ Hanoi 100000, Vietnam \\ ${ }^{6}$ Department of Physics, Hanoi Pedagogical University 2, Phuc Yen, Vinh Phuc 280000, Vietnam \\ ${ }^{7}$ Universidad Técnica Federico Santa María and Centro Científico-Tecnológico de Valparaíso, \\ Casilla 110-V, Valparaíso, Chile
}

(Received 18 April 2019; published 8 July 2019)

\begin{abstract}
We perform a comprehensive analysis of several phenomenological aspects of the renormalizable extension of the inert 3-3-1 model with sequentially loop-generated Standard Model fermion mass hierarchy. Special attention is paid to the study of the constraints arising from the experimental data on the $\rho$ parameter, as well as those ones resulting from the charged lepton flavor violating process $\mu \rightarrow e \gamma$ and dark matter. We also study the single $Z^{\prime}$ production via Drell-Yan mechanism at the LHC. We have found that $Z^{\prime}$ gauge bosons heavier than about $4 \mathrm{TeV}$ comply with the experimental constraints on the oblique $\rho$ parameter as well as with the collider constraints. In addition, we have found that the constraint on the charged lepton flavor violating decay $\mu \rightarrow e \gamma$ sets the sterile neutrino masses to be lighter than about $1.12 \mathrm{TeV}$. In addition the model allows charged lepton flavor violating processes within reach of the forthcoming experiments. The scalar potential and the gauge sector of the model are analyzed and discussed in detail. Our model successfully accommodates the observed dark matter relic density.
\end{abstract}

DOI: 10.1103/PhysRevD.100.015004

\section{INTRODUCTION}

Despite its great successes, the Standard Model (SM) does not explain the observed mass and mixing hierarchies in the fermion sector, which remain without a compelling explanation. It is known that in the SM, the masses of the matter fields are generated from the Yukawa interactions. In addition, the Cabibbo-Kobayashi-Maskawa quark mixing matrix is also constructed from the same Yukawa couplings. To solve these puzzles, some mechanisms have been proposed. To the best of our knowledge, the first attempt to explain the huge differences in the SM fermion masses is

\footnotetext{
*hoangngoclong@tdtu.edu.vn

†nvhop@ctu.edu.vn

1thue@iop.vast.vn

\$abcthao@gmail.com

"antonio.carcamo@usm.cl
}

Published by the American Physical Society under the terms of the Creative Commons Attribution 4.0 International license. Further distribution of this work must maintain attribution to the author(s) and the published article's title, journal citation, and DOI. Funded by SCOAP ${ }^{3}$. the Froggatt-Nielsen (FN) mechanism [1]. According to the FM mechanism, the mass differences between generations of fermions arise from suppression factors depending on the FN charges of the particles. It has been noticed that in order to implement the aforementioned mechanism, the effective Yukawa interactions have to be introduced, thus making this theory nonrenormalizable. From this point of view, the recent mechanism proposed by Cárcamo, Kovalenko, and Schmidt [2] (called by CKS mechanism) based on sequential loop suppression mechanism, is more natural since its suppression factor arises from the loop factor $l \approx(1 / 4 \pi)^{2}$.

One of the main purposes of the models based on the gauge group $S U(3)_{C} \times S U(3)_{L} \times U(1)_{X}$ (for short, 3-3-1 model) [3-10] is concerned with the search of an explanation for the number of generations of fermions. Combined with the QCD asymptotic freedom, the 3-3-1 models provide an explanation for the number of fermion generations. These models have nonuniversal $U(1)_{X}$ gauge assignments for the left handed quarks fields, thus implying that the cancellation of chiral anomalies is fulfilled when the number of $S U(3)_{L}$ fermionic triplets is equal to the number of $S U(3)_{L}$ fermionic antitriplets, which happens 
when the number of fermion families is a multiple of three. Some other advantages of the 3-3-1 models are: (i) they solve the electric charge quantization [11,12], (ii) they contain several sources of $C P$ violation $[13,14]$, and (iii) they have a natural Peccei-Quinn symmetry, which solves the strong- $C P$ problem [15-18].

In the framework of the 3-3-1 models, most of the research is focused on radiative seesaw mechanisms, and but some involving nonrenormalizable interactions introduced to explain the SM fermion mass and mixing pattern (see references in Ref. [19]).

The FN mechanism was implemented in the 3-3-1 models in Ref. [20]. It is interesting to note that the FN mechanism does not produce a new scale since the scale of the flavor breaking is the same as the symmetry breaking scale of the model.

The CKS mechanism has been implemented for the first time in the 3-3-1 model without exotic electric charges $(\beta=-1 / \sqrt{3})$ in Ref. [19]. The implementation of the CKS mechanism in the 3-3-1 model leads to viable renormalizable 3-3-1 model that provides a dynamical explanation for the observed SM fermion mass spectrum and mixing parameters consistent with the SM low energy fermion flavor data [19]. It is worth mentioning that the extension of the inert 331 model of Ref. [19] contains a residual discrete $Z_{2}^{\left(L_{g}\right)}$ lepton number symmetry arising from the spontaneous breaking of the global $U(1)_{L_{g}}$ symmetry. Under this residual symmetry, the leptons are charged and the other particles are neutral [19].

However, in the mentioned work, the authors have just focused on the data concerning fermions (both quarks and leptons including neutrino mass and mixing), but some questions are open for the future study.

The purpose of this work is to study several phenomenological aspects of the renormalizable extension of the inert 3-3-1 model with sequentially loop-generated SM fermion mass hierarchy. In particular, the constraints arising from the experimental data on the $\rho$ parameter, as well as those ones resulting from the charged lepton flavor violating process $\mu \rightarrow e \gamma$ and dark matter. Furthermore our work discusses the $Z^{\prime}$ production at proton-proton collider via quark-antiquark annihilation. To determine the oblique $\rho$ parameter constraints on the $S U(3)_{L} \times U(1)_{X}$ symmetry breaking scale $v_{\chi}$, which will be used to constrain the heavy $Z^{\prime}$ gauge boson mass, we proceed to study in detail the gauge and Higgs sectors of the model. In addition we determine the constraints imposed by the charged lepton flavor violating process $\mu \rightarrow e \gamma$ and dark matter on the model parameter space. In what regards the scalar potential of the model, due to the implemented symmetries, the Higgs sector is rather simple and can be completely solved. All Goldstone bosons and the SM like Higgs boson are defined.

The further content of this paper is as follows. In Sec. II, we briefly present particle content and SSB of the model.
Section III is devoted to gauge boson mass and mixing. Taking into account of data on the $\rho$ parameter, and if only contributions of the gauge bosons are mentioned, we will show that the mass of the heavy neutral boson $Z^{\prime}$ will be constrained nearly to the excluded regions derived from other experimental data such as LHC searches, and $K, D$, and $B$ meson mixing. The Higgs sector is considered in Sec. IV. The Higgs sector consists of two parts: the first part contains lepton number conserving terms and the second one is lepton number violating. We study in details the first part and show that the Higgs sector has all necessary ingredients. The $\rho$ parameter will be investigated including Higgs contributions. In Sec. V, lepton flavor violating decays of the charged leptons are discussed, where sterile neutral lepton masses are constrained. Section VI is devoted to the production of the heavy $Z^{\prime}$ and the heavy neutral scalar $H_{4}$. In Sec. VII, we deal with the DM relic density. We make conclusions in Sec. VIII. The scalar potential of the model is given in Appendix.

\section{REVIEW OF THE MODEL}

To implement the CKS mechanism, only the heaviest particles such as the exotic fermions and the top quark get masses at tree level. The next-medium ones: bottom, charm quarks, tau, and muon get masses at one-loop level. Finally, the lightest particles: up, down, strange quarks and the electron acquire masses at two-loop level. To forbid the usual Yukawa interactions, the discrete symmetries should be implemented. Hence, the full symmetry of the model under consideration is

$$
S U(3)_{C} \times S U(3)_{L} \times U(1)_{X} \times Z_{4} \times Z_{2} \times U(1)_{L_{g}},
$$

where $L_{g}$ is the generalized lepton number defined in Refs. $[19,21]$. It is interesting to note that, in this model, the light active neutrinos get their masses from a combination of linear and inverse seesaw mechanisms at twoloop level.

As in the ordinary 3-3-1 model without exotic electric charges, the quark sector contains the following $S U(3)_{C} \times$ $S U(3)_{L} \times U(1)_{X}$ representations [19]

$$
\begin{aligned}
Q_{n L} & =\left(D_{n},-U_{n}, J_{n}\right)_{L}^{T} \sim\left(3,3^{*}, 0\right), \\
Q_{3 L} & =\left(U_{3}, D_{3}, T\right)_{L}^{T} \sim\left(3,3, \frac{1}{3}\right), \quad n=1,2 \\
D_{i R} & \sim\left(3,1,-\frac{1}{3}\right), \quad U_{i R} \sim\left(3,1, \frac{2}{3}\right), \quad i=1,2,3 \\
J_{n R} & \sim\left(3,1,-\frac{1}{3}\right), \quad T_{R} \sim\left(3,1, \frac{2}{3}\right), \\
\tilde{T}_{L, R} & \sim\left(3,1, \frac{2}{3}\right), \quad B_{L, R} \sim\left(3,1,-\frac{1}{3}\right)
\end{aligned}
$$


where $\sim$ denotes the quantum numbers for the three above subgroups, respectively. Note that the $S U(3)_{L}$ singlet exotic up type quarks $\tilde{T}_{L, R}$, down type quarks $B_{L, R}$ in the last line of Eq. (2) are added to the quark spectrum of the ordinary 3-3-1 model in order to implement the CKS mechanism.
In the leptonic sector, besides the usual $S U(3)_{L}$ lepton triplets, the model contains extra three charged leptons $E_{j(L, R)}(j=1,2,3)$ and four neutral leptons, i.e., $N_{j R}$ and $\Psi_{R}(j=1,2,3)$. The leptonic fields have the following $S U(3)_{C} \times S U(3)_{L} \times U(1)_{X}$ assignments:

$$
\begin{aligned}
& L_{i L}=\left(\nu_{i}, e_{i}, \nu_{i}^{c}\right)_{L}^{T} \sim\left(1,3,-\frac{1}{3}\right), & & e_{i R} \sim(1,1,-1), \quad i=1,2,3, \\
E_{1 L} & \sim(1,1,-1), & E_{2 L} \sim(1,1,-1), & E_{3 L} \sim(1,1,-1), \\
E_{1 R} & \sim(1,1,-1), & E_{2 R} \sim(1,1,-1), & E_{3 R} \sim(1,1,-1), \\
N_{1 R} & \sim(1,1,0), & N_{2 R} \sim(1,1,0), & N_{3 R} \sim(1,1,0), \quad \Psi_{R} \sim(1,1,0),
\end{aligned}
$$

where $\nu_{i L}, \nu^{c} \equiv \nu_{R}^{c}$ and $e_{i L}\left(e_{L}, \mu_{L}, \tau_{L}\right)$ are the neutral and charged lepton families, respectively.

The Higgs sector contains three scalar triplets: $\chi, \eta$, and $\rho$ and seven singlets $\varphi_{1}^{0}, \varphi_{2}^{0}, \xi^{0}, \phi_{1}^{+}, \phi_{2}^{+}, \phi_{3}^{+}$, and $\phi_{4}^{+}$. Hence, the scalar spectrum of the model is composed of the following fields

$$
\begin{gathered}
\chi=\langle\chi\rangle+\chi^{\prime} \sim\left(1,3,-\frac{1}{3}\right), \\
\langle\chi\rangle=\left(0,0, \frac{v_{\chi}}{\sqrt{2}}\right)^{T}, \quad \chi^{\prime}=\left(\chi_{1}^{0}, \chi_{2}^{-}, \frac{1}{\sqrt{2}}\left(R_{\chi_{3}^{0}}-i I_{\chi_{3}^{0}}\right)\right)^{T}, \\
\rho=\left(\rho_{1}^{+}, \frac{1}{\sqrt{2}}\left(R_{\rho}-i I_{\rho}\right), \rho_{3}^{+}\right)^{T} \sim\left(1,3, \frac{2}{3}\right), \\
\eta=\langle\eta\rangle+\eta^{\prime} \sim\left(1,3,-\frac{1}{3}\right), \\
\langle\eta\rangle=\left(\frac{v_{\eta}}{\sqrt{2}}, 0,0\right)^{T}, \quad \eta^{\prime}=\left(\frac{1}{\sqrt{2}}\left(R_{\eta_{1}^{0}}-i I_{\eta_{1}^{0}}\right), \eta_{2}^{-}, \eta_{3}^{0}\right)^{T}, \\
\varphi_{1}^{0} \sim(1,1,0), \quad \varphi_{2}^{0} \sim(1,1,0), \quad \phi_{2}^{+} \sim(1,1,1), \quad \phi_{3}^{+} \sim(1,1,1), \quad \phi_{4}^{+} \sim(1,1,1), \\
\phi_{1}^{+} \sim(1,1,1), \quad\left\langle\xi^{0}\right\rangle=\frac{v_{\xi}}{\sqrt{2}}, \quad \xi^{0 \prime}=\frac{1}{\sqrt{2}}\left(R_{\xi^{0}}-i I_{\xi^{0}}\right) \sim(1,1,0) . \\
\xi^{0}=\left\langle\xi^{0}\right\rangle+\xi^{0 \prime}, \quad
\end{gathered}
$$

The $Z_{4} \times Z_{2}$ assignments of scalar the fields are shown in Table I.

The fields with nonzero lepton number are presented in Table II. Note that the three gauge singlet neutral leptons $N_{i R}$ as well as the elements in the third component of the lepton triplets, namely $\nu_{i L}^{c}$ have lepton number equal -1 .

In the model under consideration, the spontaneous symmetry breaking (SSB) occurs by two steps [19]. The first step is triggered by the vacuum expectation values

TABLE I. Scalar assignments under $Z_{4} \times Z_{2}$.

\begin{tabular}{ccccccccccc}
\hline \hline & $\chi$ & $\eta$ & $\rho$ & $\varphi_{1}^{0}$ & $\varphi_{2}^{0}$ & $\phi_{1}^{+}$ & $\phi_{2}^{+}$ & $\phi_{3}^{+}$ & $\phi_{4}^{+}$ & $\xi^{0}$ \\
\hline$Z_{4}$ & 1 & 1 & -1 & -1 & $i$ & $i$ & -1 & -1 & 1 & 1 \\
$Z_{2}$ & -1 & -1 & 1 & 1 & 1 & 1 & 1 & -1 & -1 & 1 \\
\hline \hline
\end{tabular}

(VEVs) of the $\chi_{3}^{0}$ and $\xi^{0}$ scalar fields. At this step, all new extra fermions, non-SM gauge bosons, as well as the electrically neutral gauge singlet lepton $\Psi_{R}$ gain masses. In addition, the entries of the neutral lepton mass matrices with negative lepton number $(-1)$ also get values proportional to $v_{\xi}$. At this step, the initial group breaks down to the direct product of the SM gauge group and the $Z_{4} \times$ $Z_{2}^{\left(L_{g}\right)}$ discrete group. The second step is triggered by $v_{\eta}$ providing masses for the top quark as well as for the $W$ and $Z$ gauge bosons and leaving the $S U(3)_{C} \times U(1)_{Q} \times Z_{4} \times$ $Z_{2}^{\left(L_{g}\right)}$ symmetry preserved. Here $Z_{2}^{\left(L_{g}\right)}$ is residual symmetry where only leptons are charged, thus forbidding interactions having an odd number of leptons. This is crucial to guarantee the proton stability [19]. Thus 
TABLE II. Nonzero lepton number $L$ of fields.

\begin{tabular}{cccccccccccccccccc}
\hline \hline & $T_{L, R}$ & $J_{1 L, R}$ & $J_{2 L, R}$ & $\nu_{i L}^{c}$ & $e_{i L, R}$ & $E_{i L, R}$ & $N_{i R}$ & $\Psi_{R}$ & $\chi_{1}^{0}$ & $\chi_{2}^{+}$ & $\eta_{3}^{0}$ & $\rho_{3}^{+}$ & $\phi_{2}^{+}$ & $\phi_{3}^{+}$ & $\phi_{4}^{+}$ & $\xi^{0}$ & $i=1,2,3$ \\
\hline$L$ & -2 & 2 & 2 & -1 & 1 & 1 & -1 & 1 & 2 & 2 & -2 & -2 & -2 & -2 & -2 & -2 & \\
\hline \hline
\end{tabular}

$$
\begin{aligned}
& S U(3)_{C} \times S U(3)_{L} \times U(1)_{X} \times Z_{4} \times Z_{2} \times U(1)_{L_{g}} \\
& \stackrel{v_{\chi}, v_{\xi}}{\longrightarrow} S U(3)_{C} \times S U(2)_{L} \times U(1)_{Y} \times Z_{4} \times Z_{2}^{\left(L_{g}\right)} \\
& \stackrel{v_{\eta}}{\longrightarrow} S U(3)_{C} \times U(1)_{Q} \times Z_{4} \times Z_{2}^{\left(L_{g}\right)} .
\end{aligned}
$$

A consequence of the chain in (7) is

$$
v_{\eta}=v=246 \mathrm{GeV} \ll v_{\chi} \sim v_{\xi} \sim \mathcal{O}(10) \mathrm{TeV} .
$$

The corresponding Majoron associated to the spontaneous breaking of the $U(1)_{L_{q}}$ global symmetry is a gaugesinglet scalar and, therefore, unobservable.

An explanation for the relation $v_{\chi} \sim v_{\xi} \sim \mathcal{O}(10) \mathrm{TeV}$ is provided in the following. The present lower limits on the $Z^{\prime}$ gauge boson mass in 3-3-1 models arising from LHC searches reach around $2.5 \mathrm{TeV}$ [22]. These bounds can be translated into limits of about $6.3 \mathrm{TeV}$ on the $S U(3)_{C} \times$ $S U(3)_{L} \times U(1)_{X}$ gauge symmetry breaking scale $v_{\chi}$. Furthermore, electroweak data from the decays $B_{s, d} \rightarrow$ $\mu^{+} \mu^{-}$and $B_{d} \rightarrow K^{*}(K) \mu^{+} \mu^{-}$set lower bounds on the $Z^{\prime}$ gauge boson mass ranging from $1 \mathrm{TeV}$ up to $3 \mathrm{TeV}$ [23-27]. Furthermore, as shown in Ref. [28], the experimental data on $K, D$, and $B$ meson mixings set a lower bound of about $4 \mathrm{TeV}$ for the $Z^{\prime}$ gauge boson mass in 3-3-1 models, which translates in a lower limit of about $10 \mathrm{TeV}$ for the $S U(3)_{L} \times$ $U(1)_{X}$ gauge symmetry breaking scale $v_{\chi}$.

Finally, to close this section we provide a justification of the role of the different particles of our model:

(1) The presence of the $S U(3)_{L}$ scalar singlet $\phi_{3}^{+}$, is needed to generate two loop level down and strange quark masses, as shown in Ref. [19]. Besides that, in order to implement a two loop level radiative seesaw mechanism for the generation of the up, down, and strange quark masses as well as the electron mass, the $Z_{4}$ charged $S U(3)_{L}$ scalar singlets $\varphi_{1}^{0}, \varphi_{2}^{0}, \phi_{1}^{+}$, $\phi_{2}^{+}$(which do not acquire a vacuum expectation value) are also required in the scalar sector. The $Z_{4}$ charged $S U(3)_{L}$ scalar singlet $\varphi_{1}^{0}$ is also needed to generate one loop level masses for the charm and bottom quarks as well as for the tau and muon leptons. The $Z_{4}$ charged $S U(3)_{L}$ scalar singlets $\varphi_{2}^{0}$ and $\phi_{3}^{+}$as well as the $S U(3)_{L}$ scalar singlet $\phi_{4}^{+}$, neutral under $Z_{4}$ are also crucial for the implementation of two loop level linear and inverse seesaw mechanisms that give rise to the light active neutrino masses. The $S U(3)_{L}$ scalar singlet $\xi^{0}$ is introduced to spontaneously break the $U(1)_{L_{g}}$ generalized lepton number symmetry and thus giving rise to a tree-level mass for the right handed Majorana neutrino $\Psi_{R}$. It is crucial for generating two looplevel masses for the down and strange quarks.

(2) The $S U(3)_{L}$ singlet exotic down type quark, i.e., $B$, is crucial for the implementation of the one loop level radiative seesaw mechanism that generate the bottom quark mass. The $S U(3)_{L}$ singlet exotic up type quarks, i.e., $\tilde{T}_{1}$ and $\tilde{T}_{2}$, are needed to generate a one loop level charm quark mass as well as two loop level down and strange quark masses. The three $S U(3)_{L}$ singlet exotic charged leptons, i.e., $E_{j}$ $(j=1,2,3)$, are required in order to provide the radiative seesaw mechanisms that generate one loop level tau and muon masses and two loop level electron mass. The four right handed Majorana neutrinos, i.e., $N_{j R}(j=1,2,3), \Psi_{R}$, are crucial for the implementation of the two loop level linear and inverse seesaw mechanisms that give rise to the light active neutrino masses.

\section{GAUGE BOSONS}

\section{A. Gauge boson masses and mixing}

After SSB, the gauge bosons get masses arising from the kinetic terms for the $\eta$ and $\chi S U(3)_{L}$ scalar triplets, as follows:

$$
L_{\text {mass }}^{\text {gauge }}=\left(D_{\mu}\langle\chi\rangle\right)^{\dagger} D^{\mu}\langle\chi\rangle+\left(D_{\mu}\langle\eta\rangle\right)^{\dagger} D^{\mu}\langle\eta\rangle,
$$

with the covariant derivative for triplet defined as

$$
D_{\mu}=\partial_{\mu}-i g A_{\mu a} \frac{\lambda_{a}}{2}-i g_{X} X \frac{\lambda_{9}}{2} B_{\mu}
$$

where $g$ and $g_{X}$ are the gauge coupling constants of the $S U(3)_{L}$ and $U(1)_{X}$ groups, respectively. Here, $\lambda_{9}=$ $\sqrt{2 / 3} \operatorname{diag}(1,1,1)$ is defined such that $\operatorname{Tr}\left(\lambda_{9} \lambda_{9}\right)=2$, similarly as the usual Gell-Mann matrix $\lambda_{a}, a=1$, $2,3, \ldots, 8$. By matching gauge the coupling constants at the $S U(3)_{L} \times U(1)_{X}$ symmetry breaking scale, the following relation is obtained [9]

$$
t \equiv \frac{g_{X}}{g}=\frac{3 \sqrt{2} \sin \theta_{W}\left(M_{Z^{\prime}}\right)}{\sqrt{3-4 \sin ^{2} \theta_{W}\left(M_{Z^{\prime}}\right)}} .
$$

Let us provide the definition of the Weinberg angle $\theta_{W}$. As in the SM, one puts $g^{\prime}=g \tan \theta_{W}$, where $g^{\prime}$ is gauge coupling of the $U(1)_{Y}$ subgroup satisfying the relation [9] 


$$
g^{\prime}=\frac{\sqrt{3} g g_{X}}{\sqrt{18 g^{2}-g_{X}^{2}}} .
$$

Thus

$$
\tan \theta_{W}=\frac{\sqrt{3} g_{X}}{\sqrt{18 g^{2}-g_{X}^{2}}} .
$$

Denoting

$$
\begin{aligned}
W_{\mu}^{ \pm} & =\frac{1}{\sqrt{2}}\left(A_{\mu 1} \mp i A_{\mu 2}\right), \quad Y_{\mu}^{ \pm}=\frac{1}{\sqrt{2}}\left(A_{\mu 6} \pm i A_{\mu 7}\right), \\
X_{\mu}^{0} & =\frac{1}{\sqrt{2}}\left(A_{\mu 4}-i A_{\mu 5}\right),
\end{aligned}
$$

and substituting (10) and (14) into (9) one gets the following squared masses for the charged/non-Hermitian gauge bosons

$$
m_{W}^{2}=\frac{g^{2}}{4} v_{\eta}^{2}, \quad M_{X^{0}}^{2}=\frac{g^{2}}{4}\left(v_{\chi}^{2}+v_{\eta}^{2}\right), \quad M_{Y}^{2}=\frac{g^{2}}{4} v_{\chi}^{2},
$$

where $v_{\eta}=v=246 \mathrm{GeV}$, as expected.

From Eq. (15) we find the following gauge boson mass squared splitting

$$
M_{X^{0}}^{2}-M_{Y}^{2}=m_{W}^{2}
$$

For neutral gauge bosons, the squared mass mixing matrix has the form

$$
L_{\text {mass }}^{\text {ngauge }}=\frac{1}{2} V^{T} M_{\text {ngauge }}^{2} V
$$

where $V^{T}=\left(A_{\mu 3}, A_{\mu 8}, B_{\mu}\right)$ and

$$
M_{\text {ngauge }}^{2}=\frac{g^{2}}{4}\left(\begin{array}{ccc}
v_{\eta}^{2} & \frac{v_{\eta}^{2}}{\sqrt{3}} & -\frac{2 t}{3 \sqrt{6}} v_{\eta}^{2} \\
& \frac{1}{3}\left(4 v_{\chi}^{2}+v_{\eta}^{2}\right) & \frac{2 t}{9 \sqrt{2}}\left(2 v_{\chi}^{2}-v_{\eta}^{2}\right) \\
& & \frac{2 t^{2}}{27}\left(v_{\chi}^{2}+v_{\eta}^{2}\right)
\end{array}\right)
$$

The down-left entries in (18) are not written, due to the fact that the above matrix is symmetric.

The matrix in (18) has vanishing determinant, thus giving rise to a massless gauge boson, which corresponds to the photon. The diagonalization of the squared mass matrix for neutral gauge bosons of Eq. (18) is divided in two steps. In the first step, the massive fields are identified as

$$
\begin{aligned}
& A_{\mu}=s_{W} A_{\mu 3}+c_{W}\left(-\frac{t_{W}}{\sqrt{3}} A_{\mu 8}+\sqrt{1-\frac{t_{W}^{2}}{3}} B_{\mu}\right), \\
& Z_{\mu}=c_{W} A_{\mu 3}-s_{W}\left(-\frac{t_{W}}{\sqrt{3}} A_{\mu 8}+\sqrt{1-\frac{t_{W}^{2}}{3}} B_{\mu}\right), \\
& Z_{\mu}^{\prime}=\sqrt{1-\frac{t_{W}^{2}}{3}} A_{\mu 8}+\frac{t_{W}}{\sqrt{3}} B_{\mu},
\end{aligned}
$$

where we have denoted $s_{W}=\sin \theta_{W}, c_{W}=\cos \theta_{W}, t_{W}=$ $\tan \theta_{W}$. The coupling of the photon $A_{\mu}$ gives $e=g s_{W}$. After the first step, the squared mass matrix is a block diagonal one in the new basis $\left(A_{\mu}, Z_{\mu}, Z_{\mu}^{\prime}\right)$, where the entry in the top is zero (due to the masslessness of the photon), while the $2 \times 2$ matrix for $\left(Z_{\mu}, Z_{\mu}^{\prime}\right)$ in the bottom has the form

$$
M_{(2 \times 2)}^{2}=\left(\begin{array}{cc}
M_{Z}^{2} & M_{Z Z^{\prime}}^{2} \\
M_{Z Z^{\prime}}^{2} & M_{Z^{\prime}}^{2}
\end{array}\right) .
$$

The matrix elements in (20) are given by

$$
\begin{aligned}
M_{Z}^{2} & =\frac{g^{2} v_{\eta}^{2}}{4 c_{W}^{2}}=\frac{m_{W}^{2}}{c_{W}^{2}}, \\
M_{Z Z^{\prime}}^{2} & =\frac{g^{2}}{4 c_{W}^{2} \sqrt{3-4 s_{W}^{2}}} v_{\eta}^{2}\left(1-2 s_{W}^{2}\right), \\
M_{Z^{\prime}}^{2} & =\frac{g^{2} c_{W}^{2}}{4\left(3-4 s_{W}^{2}\right)}\left[4 v_{\chi}^{2}+\frac{v_{\eta}^{2}\left(1-2 s_{W}^{2}\right)^{2}}{c_{W}^{4}}\right] .
\end{aligned}
$$

Note that our formula of $M_{Z^{\prime}}^{2}$ is consistent with that given in [23].

In the last step of diagonalization, the $Z-Z^{\prime}$ mixing angle $\phi$ and mass eigenstates $Z^{1,2}$ are determined as

$$
\begin{gathered}
\tan 2 \phi=\frac{2 M_{Z Z^{\prime}}^{2}}{M_{Z^{\prime}}^{2}-M_{Z}^{2}}, \\
Z_{\mu}^{1}=Z_{\mu} \cos \phi-Z_{\mu}^{\prime} \sin \phi, \\
Z_{\mu}^{2}=Z_{\mu} \sin \phi+Z_{\mu}^{\prime} \cos \phi .
\end{gathered}
$$

Our definition of $\phi$ is consistent with that in Ref. [29] needed to study the $\rho$ parameter.

The masses of physical neutral gauge bosons are determined as

$$
\begin{aligned}
& M_{Z^{1}}^{2}=\frac{1}{2}\left\{M_{Z^{\prime}}^{2}+M_{Z}^{2}-\left[\left(M_{Z^{\prime}}^{2}-M_{Z}^{2}\right)^{2}+4\left(M_{Z Z^{\prime}}^{2}\right)^{2}\right]^{\frac{1}{2}}\right\}, \\
& M_{Z^{2}}^{2}=\frac{1}{2}\left\{M_{Z^{\prime}}^{2}+M_{Z}^{2}+\left[\left(M_{Z^{\prime}}^{2}-M_{Z}^{2}\right)^{2}+4\left(M_{Z Z^{\prime}}^{2}\right)^{2}\right]^{\frac{1}{2}}\right\} .
\end{aligned}
$$

In the limit $v_{\chi}^{2} \gg v_{\eta}^{2}$, one approximates

$$
M_{Z^{1}}^{2} \simeq M_{Z}^{2}-\frac{\left(M_{Z Z^{\prime}}^{2}\right)^{2}}{M_{Z^{\prime}}^{2}}+M_{Z}^{2} \times \mathcal{O}\left(\frac{v_{\eta}^{4}}{v_{\chi}^{4}}\right),
$$



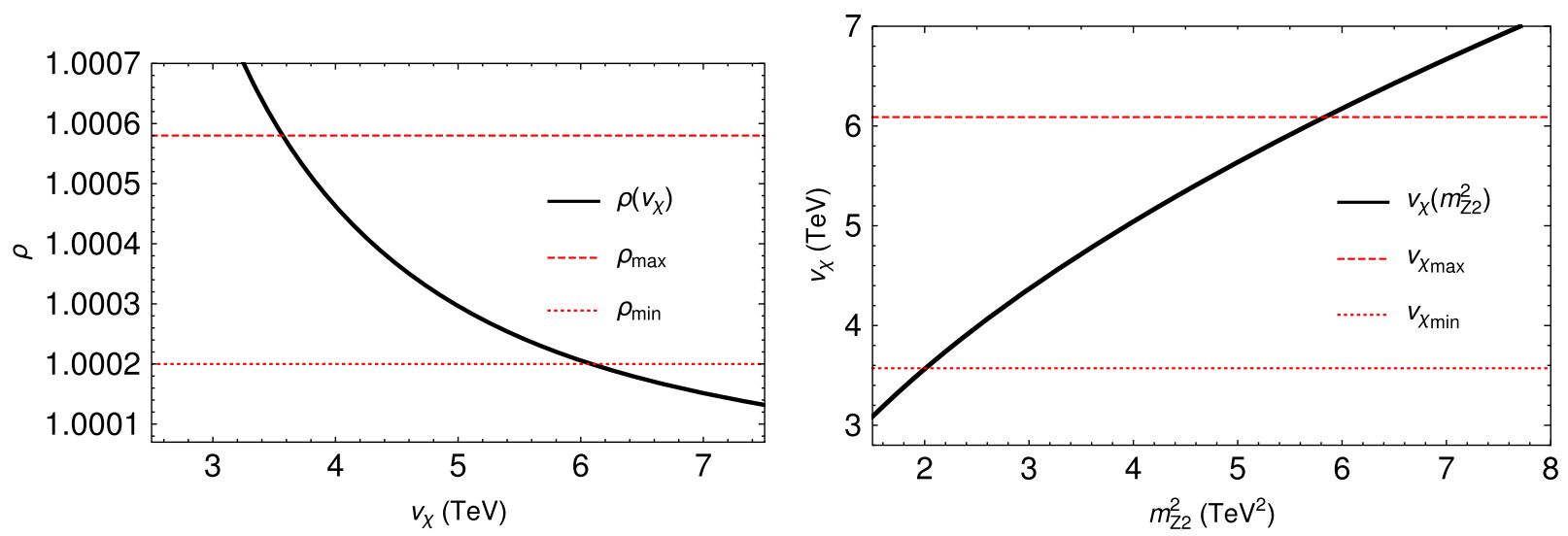

FIG. 1. Left-panel: $\rho$ parameter as a function of $v_{\chi}$, upper and lower horizontal lines are upper and lower limits in (31). Right-panel: Relation between $v_{\chi}$ and $M_{Z_{2}}^{2}$, upper horizontal lines are an upper and a lower limits of $v_{\chi}$, respectively.

$$
\begin{gathered}
M_{Z^{2}}^{2} \simeq M_{Z^{\prime}}^{2}+\frac{\left(M_{Z Z^{\prime}}^{2}\right)^{2}}{M_{Z^{\prime}}^{2}}+M_{Z}^{2} \times \mathcal{O}\left(\frac{v_{\eta}^{4}}{v_{\chi}^{4}}\right) \simeq M_{Z^{\prime}}^{2} \\
\tan \phi \simeq \frac{\left(1-2 s_{W}^{2}\right) \sqrt{3-4 s_{W}^{2}}}{4 c_{W}^{4}}\left(\frac{v_{\eta}^{2}}{v_{\chi}^{2}}\right) .
\end{gathered}
$$

\section{B. Limit on $Z^{\prime}$ mass from the $\rho$ parameter}

The presence of the non-SM particles modifies the oblique corrections of the SM, the values of which have been extracted from high precision experiments.
Consequently, the validity of our model depends on the condition that the non-SM particles do not contradict those experimental results. Let us note that one of the most important observables in the SM is the $\rho$ parameter defined as

$$
\rho=\frac{m_{W}^{2}}{c_{W}^{2} M_{Z}^{2}} .
$$

For the model under consideration, one-loop contributions of the new heavy gauge bosons to the oblique correction lead to the following form of the $\rho$ parameter [29]

$$
\begin{gathered}
\rho-1 \simeq \tan ^{2} \phi\left(\frac{M_{Z^{\prime}}^{2}}{m_{Z}^{2}}-1\right)+\frac{3 \sqrt{2} G_{F}}{16 \pi^{2}}\left[M_{+}^{2}+M_{0}^{2}+\frac{2 M_{+}^{2} M_{0}^{2}}{M_{+}^{2}-M_{0}^{2}} \ln \frac{M_{0}^{2}}{M_{+}^{2}}\right] \\
-\frac{\alpha\left(m_{Z}\right)}{4 \pi s_{W}^{2}}\left[t_{W}^{2} \ln \frac{M_{0}^{2}}{M_{+}^{2}}+\frac{\varepsilon^{2}\left(M_{+}, M_{0}\right)}{2}+O\left(\varepsilon^{3}\left(M_{+}, M_{0}\right)\right)\right],
\end{gathered}
$$

where $M_{0}=M_{X^{0}}, M_{+}=M_{Y^{+}}$and $\varepsilon(M, m) \equiv \frac{M^{2}-m^{2}}{m^{2}}$.

Combining with Eq. (16), one gets

$$
\begin{aligned}
\rho-1 \simeq & \tan ^{2} \phi\left(\frac{M_{Z^{\prime}}^{2}}{m_{Z}^{2}}-1\right)+\frac{3 \sqrt{2} G_{F}}{16 \pi^{2}}\left[2 M_{Y^{+}}^{2}+m_{W}^{2}-\frac{2 M_{Y^{+}}^{2}\left(M_{Y^{+}}^{2}+m_{W}^{2}\right)}{m_{W}^{2}} \ln \frac{\left(M_{Y^{+}}^{2}+m_{W}^{2}\right)}{M_{Y^{+}}^{2}}\right] \\
& -\frac{\alpha\left(m_{Z}\right)}{4 \pi s_{W}^{2}}\left[t_{W}^{2} \ln \frac{\left(M_{Y^{+}}^{2}+m_{W}^{2}\right)}{M_{Y^{+}}^{2}}+\frac{m_{W}^{4}}{2\left(M_{Y^{+}}^{2}+m_{W}^{2}\right)^{2}}\right]
\end{aligned}
$$

where $\alpha\left(m_{Z}\right) \approx \frac{1}{128}[30]$.

Taking into account $s_{W}^{2}=0.23122[30]$ and

$$
\rho=1.00039 \pm 0.00019
$$

we have plotted $\Delta \rho$ as a function of $v_{\chi}$ in Fig. 1 (the leftpanel). From Fig. 1 (the left-panel), it follows

$$
3.57 \mathrm{TeV} \leq v_{\chi} \leq 6.09 \mathrm{TeV} .
$$

Substituting (32) into (26) and evaluating in Fig. 1 (the right-panel) we get a bound on the $Z^{\prime}$ mass as follows

$$
1.42 \mathrm{TeV} \leq M_{Z^{2}} \leq 2.42 \mathrm{TeV} .
$$

Then, the bilepton gauge boson mass is constrained to be in the range: 


$$
465 \mathrm{GeV} \leq M_{Y} \leq 960 \mathrm{GeV},
$$

where $m_{W}=80.379 \mathrm{GeV}$ [30]. The above limit is stronger than the one obtained from the wrong muon decay $M_{Y} \geq$ $230 \mathrm{GeV}$ [31].

It is worth mentioning that the second term in (30) is much smaller the first one. Consequently, the limit derived from the tree level contribution is very close to the one obtained when we consider the radiative corrections arising from heavy vector exchange.

From LHC searches, it follows that the lower bound on the $Z^{\prime}$ boson mass in 3-3-1 models ranges from 2.5 to $3 \mathrm{TeV}$ [22,32]. From the decays $B_{s, d} \rightarrow \mu^{+} \mu^{-}$and $B_{d} \rightarrow$ $K^{*}(K) \mu^{+} \mu^{-}$[23-27], the lower limit on the $Z^{\prime}$ boson mass ranges from $1 \mathrm{TeV}$ to $3 \mathrm{TeV}$. We will show that when scalar contributions to the $\rho$ parameter are included, there will exist allowed $m_{Z_{2}}$ values larger than the range given in (33), so that they satisfy the recent lower bounds concerned from LHC searches.

For conventional notation, hereafter we will call $Z^{1}$ and $Z^{2}$ by $Z$ and $Z^{\prime}$, respectively.

Now we turn to the main subject- the Higgs sector.

\section{ANALYSIS OF THE LEPTON NUMBER CONSERVING PART OF THE SCALAR POTENTIAL}

Below we present lepton number conserving part $V_{\mathrm{LNC}}$ of the scalar potential of the model shown in Appendix. Expanding the Higgs potential around the VEVs, one gets

$$
M_{\text {charged }}^{2}=\left(\begin{array}{c}
A+\frac{1}{2} v_{\eta}^{2}\left(\lambda_{6}+\lambda_{9}\right) \\
0 \\
\frac{1}{2} v_{\eta} v_{\xi} \lambda_{3}
\end{array}\right.
$$

where we have used the following notations

$$
\begin{aligned}
A & \equiv \mu_{\rho}^{2}+\frac{1}{2}\left[v_{\chi}^{2} \lambda_{18}+\lambda_{\rho \xi} v_{\xi}^{2}\right], \\
B_{i} & \equiv \frac{1}{2}\left(v_{\chi}^{2} \lambda_{i}^{\chi \phi}+v_{\eta}^{2} \lambda_{i}^{\eta \phi}+v_{\xi}^{2} \lambda_{i}^{\phi \xi}\right), \quad i=1,2,3,4 .
\end{aligned}
$$

From (38), it follows that in the limit $v_{\eta} \ll v_{\xi}, \rho_{1}^{+}$is a physical field with mass

$$
m_{\rho_{1}^{+}}^{2}=A+\frac{1}{2} v_{\eta}^{2}\left(\lambda_{6}+\lambda_{9}\right)
$$

while the two massive bilepton scalars $\rho_{3}^{+}$and $\phi_{3}^{+}$mix with each other.

Now we turn into $C P$-odd Higgs sector. There are three massless fields: $I_{\chi_{3}^{0}}, I_{\eta_{1}^{0}}$, and $I_{\xi^{0}}$. The field $I_{\varphi_{2}}$ is a physical state itself with squared mass the scalar potential minimization conditions at tree level as follows

$$
\begin{gathered}
w_{3}=0, \\
-\mu_{\chi}^{2}=v_{\chi}^{2} \lambda_{13}+\frac{1}{2} v_{\eta}^{2} \lambda_{5}+\frac{1}{2} \lambda_{\chi \xi} v_{\xi}^{2}, \\
-\mu_{\eta}^{2}=v_{\eta}^{2} \lambda_{17}+\frac{1}{2} v_{\chi}^{2} \lambda_{5}+\frac{1}{2} \lambda_{\eta \xi} v_{\xi}^{2}, \\
-\mu_{\xi}^{2}=\frac{1}{2} \lambda_{\chi \xi} v_{\chi}^{2}+\frac{1}{2} \lambda_{\eta \xi} v_{\eta}^{2}+\lambda_{\xi} v_{\xi}^{2} .
\end{gathered}
$$

From the analysis of the scalar potential, taking into account the constraint conditions of Eq. (35), we find that the charged scalar sector is composed of two massless fields, i.e., $\eta_{2}^{+}$and $\chi_{2}^{+}$which are the Goldstone bosons eaten by the longitudinal components of the $W^{+}$and $Y^{+}$gauge bosons, respectively. The other massive electrically charged fields are $\phi_{1}^{+}, \phi_{2}^{+}$and $\phi_{4}^{+}$whose masses are respectively given by:

$$
\begin{aligned}
& m_{\phi_{1}^{+}}^{2}=\mu_{\phi_{1}^{+}}^{2}+\frac{1}{2}\left[v_{\chi}^{2} \lambda_{1}^{\chi \phi}+v_{\eta}^{2} \lambda_{1}^{\eta \phi}+v_{\xi}^{2} \lambda_{1}^{\phi \xi}\right], \\
& m_{\phi_{2}^{+}}^{2}=\mu_{\phi_{2}^{+}}^{2}+\frac{1}{2}\left[v_{\chi}^{2} \lambda_{2}^{\chi \phi}+v_{\eta}^{2} \lambda_{2}^{\eta \phi}+v_{\xi}^{2} \lambda_{2}^{\phi \xi}\right], \\
& m_{\phi_{4}^{+}}^{2}=\mu_{\phi_{4}^{+}}^{2}+\frac{1}{2}\left[v_{\chi}^{2} \lambda_{4}^{\chi \phi}+v_{\eta}^{2} \lambda_{4}^{\eta \phi}+v_{\xi}^{2} \lambda_{4}^{\phi \xi}\right] .
\end{aligned}
$$

In addition, the basis $\left(\rho_{1}^{+}, \rho_{3}^{+}, \phi_{3}^{+}\right)$corresponds to the following squared mass matrix

$$
\left.\begin{array}{cc}
0 & \frac{1}{2} v_{\eta} v_{\xi} \lambda_{3} \\
A+\frac{1}{2}\left(v_{\chi}^{2} \lambda_{7}+v_{\eta}^{2} \lambda_{6}\right) & \frac{1}{\sqrt{2}} v_{\chi} w_{2} \\
\frac{1}{\sqrt{2}} v_{\chi} w_{2} & \mu_{\phi_{3}^{+}}^{2}+B_{3}
\end{array}\right),
$$

$$
m_{I_{\varphi_{2}}}^{2}=\mu_{\varphi_{2}}^{2}+B_{2}^{\prime}
$$

where

$$
B_{n}^{\prime} \equiv \frac{1}{2}\left(v_{\chi}^{2} \lambda_{n}^{\chi \varphi}+v_{\eta}^{2} \lambda_{n}^{\eta^{\varphi}}+v_{\xi}^{2} \lambda_{n}^{\varphi \xi}\right), \quad n=1,2 .
$$

The squared mass matrix of the four remaining $C P$-odd Higgs fields separate into two block diagonal submatrices corresponding to the two original base $\left(I_{\chi_{1}^{0}}, I_{\eta_{3}^{0}}\right)$ and $\left(I_{\varphi_{1}}, I_{\rho}\right)$, namely

$$
\begin{aligned}
m_{C \text { odd } 1}^{2} & =\frac{\lambda_{8}}{2}\left(\begin{array}{cc}
v_{\eta}^{2} & -v_{\chi} v_{\eta} \\
-v_{\chi} v_{\eta} & v_{\chi}^{2}
\end{array}\right), \\
m_{C \text { odd } 2}^{2} & =\left(\begin{array}{cc}
\mu_{\varphi_{1}}^{2}+B_{1}^{\prime}-C & \frac{1}{2} v_{\chi} v_{\eta}\left(\lambda_{1}-\lambda_{2}\right) \\
\frac{1}{2} v_{\chi} v_{\eta}\left(\lambda_{1}-\lambda_{2}\right) & A+\frac{\lambda_{6}}{2} v_{\eta}^{2}
\end{array}\right),
\end{aligned}
$$


where

$$
C \equiv v_{\chi}^{2} \lambda_{22}+v_{\eta}^{2} \lambda_{24}+v_{\xi}^{2} \lambda_{25}
$$

The first matrix in (43) provides two mass eigenstates, where one of them is massless,

$$
\begin{aligned}
& G_{1}=\cos \theta_{a} I_{\chi_{1}^{0}}+\sin \theta_{a} I_{\eta_{3}^{0}}, \quad m_{G_{1}}=0, \\
& A_{1}=-\sin \theta_{a} I_{\chi_{1}^{0}}+\cos \theta_{a} I_{\eta_{3}^{0}}, \quad m_{A_{1}}^{2}=\frac{\lambda_{8} v_{\chi}^{2}}{2 \cos ^{2} \theta_{a}},
\end{aligned}
$$

where

$$
\tan \theta_{a}=\frac{v_{\eta}}{v_{\chi}}
$$

The physical states relating to the second matrix in (43) are

$$
\left(\begin{array}{l}
A_{2} \\
A_{3}
\end{array}\right)=\left(\begin{array}{cc}
\cos \theta_{\rho} & \sin \theta_{\rho} \\
-\sin \theta_{\rho} & \cos \theta_{\rho}
\end{array}\right)\left(\begin{array}{c}
I_{\varphi_{1}} \\
I_{\rho}
\end{array}\right)
$$

where the mixing angle is given by

$$
\tan 2 \theta_{\rho}=\frac{v_{\chi} v_{\eta}\left(\lambda_{1}-\lambda_{2}\right)}{\left(\mu_{\varphi_{1}}^{2}-C+B_{1}^{\prime}-A-\frac{\lambda_{6}}{2} v_{\eta}^{2}\right)} .
$$

Their squared masses are

$$
m_{A_{2,3}}^{2}=\frac{1}{2}\left\{A+D_{1} \mp \sqrt{\left(A-D_{1}\right)^{2}+v_{\eta}^{2}\left[2\left(A-D_{1}\right) \lambda_{6}+v_{\eta}^{2} \lambda_{6}^{2}+v_{\chi}^{2}\left(\lambda_{13}-\lambda_{14}\right)^{2}\right]}\right\},
$$

where $D_{1}=\mu_{\varphi_{1}}^{2}+B_{1}^{\prime}-C+\frac{1}{2} v_{\eta}^{2} \lambda_{6}$.

Next, the $C P$-even scalar sector is our task. We find that $R_{\varphi_{2}}$ is physical with mass

$$
m_{R_{\varphi_{2}}}^{2}=m_{I_{\varphi_{2}}}^{2}=\mu_{\varphi_{2}}^{2}+\frac{1}{2}\left(v_{\chi}^{2} \lambda_{2}^{\chi \varphi}+v_{\eta}^{2} \lambda_{2}^{\eta \varphi}+v_{\xi}^{2} \lambda_{2}^{\varphi \xi}\right) .
$$

As mentioned in Ref. [19], the lightest scalar $\varphi_{2}^{0}$ is a possible DM candidate with light mass smaller than $1 \mathrm{TeV}$. Therefore, Eq. (50) suggests a reasonable assumption

$$
\mu_{\varphi_{2}}^{2}=-\frac{1}{2}\left(v_{\chi}^{2} \lambda_{2}^{\chi \varphi}+v_{\xi}^{2} \lambda_{2}^{\varphi \xi}\right)
$$

In this case, the model contains the complex scalar DM $\varphi_{2}^{0}$ with mass $m_{R_{\varphi_{2}}}^{2}=m_{I_{\varphi_{2}}}^{2}=\frac{1}{2} \lambda_{2}^{\eta \varphi} v_{\eta}^{2}$.

There are other seven $C P$-even Higgs components which the squared mass matrix separates into two $2 \times 2$ and one $3 \times 3$ independent matrices. The $2 \times 2$ matrices are

$$
\begin{aligned}
& m_{C \text { even } 1}^{2}=\frac{\lambda_{8}}{2}\left(\begin{array}{cc}
v_{\eta}^{2} & v_{\chi} v_{\eta} \\
v_{\chi} v_{\eta} & v_{\chi}^{2}
\end{array}\right), \\
& m_{C \text { even } 2}^{2}=\left(\begin{array}{cc}
A+\frac{\lambda_{6}}{2} v_{\eta}^{2} & -\frac{1}{2} v_{\chi} v_{\eta}\left(\lambda_{1}+\lambda_{2}\right) \\
-\frac{1}{2} v_{\chi} v_{\eta}\left(\lambda_{1}+\lambda_{2}\right) & \mu_{\varphi_{1}}^{2}+C+B_{1}^{\prime}
\end{array}\right),
\end{aligned}
$$

corresponding to the two original base $\left(R_{\chi_{1}^{0}}, R_{\eta_{3}^{0}}\right)$ and $\left(R_{\rho}, R_{\varphi_{1}}\right)$, respectively. The physical states of the first matrix in (52) are determined as follows,

$$
\begin{aligned}
R_{G_{1}} & =\cos \theta_{a} R_{\chi_{1}^{0}}+\sin \theta_{a} R_{\eta_{3}^{0}}, \quad m_{R_{G_{1}}}=0, \\
H_{1} & =-\sin \theta_{a} R_{\chi_{1}^{0}}+\cos \theta_{a} R_{\eta_{3}^{0}}, \quad m_{H_{1}}^{2}=m_{A_{1}}^{2}=\frac{\lambda_{8} v_{\chi}^{2}}{2 \cos ^{2} \theta_{a}} .
\end{aligned}
$$

The physical states of the second matrix in (52) are

$$
\left(\begin{array}{l}
H_{2} \\
H_{3}
\end{array}\right)=\left(\begin{array}{cc}
\cos \theta_{r} & \sin \theta_{r} \\
-\sin \theta_{r} & \cos \theta_{r}
\end{array}\right)\left(\begin{array}{c}
R_{\rho} \\
R_{\varphi_{1}}
\end{array}\right)
$$

where the mixing angle is

$$
\tan 2 \theta_{r}=\frac{v_{\chi} v_{\eta}\left(\lambda_{1}+\lambda_{2}\right)}{\left(\mu_{\varphi_{1}}^{2}+C+B_{1}^{\prime}-A-\frac{\lambda_{6}}{2} v_{\eta}^{2}\right)}
$$

and their squared masses are

$$
m_{H_{2,3}}^{2}=\frac{1}{2}\left\{A+D_{2} \mp \sqrt{\left(A-D_{2}\right)^{2}+v_{\eta}^{2}\left[2\left(A-D_{2}\right) \lambda_{6}+v_{\eta}^{2} \lambda_{6}^{2}+v_{\chi}^{2}\left(\lambda_{13}+\lambda_{14}\right)^{2}\right]}\right\},
$$

where $D_{2}=\mu_{\varphi_{1}}^{2}+B_{1}^{\prime}+C+\frac{1}{2} v_{\eta}^{2} \lambda_{6}$. 
The squared mass matrix corresponding to the basis $\left(R_{\chi_{3}^{0}}, R_{\eta_{1}^{0}}, R_{\xi^{0}}\right)$ is

$$
m_{C P \text { even } 3}^{2}=\left(\begin{array}{ccc}
2 v_{\chi}^{2} \lambda_{13} & v_{\chi} v_{\eta} \lambda_{5} & \lambda_{\chi \xi} v_{\chi} v_{\xi} \\
v_{\chi} v_{\eta} \lambda_{5} & 2 v_{\eta}^{2} \lambda_{17} & \lambda_{\eta \xi} v_{\eta} v_{\xi} \\
\lambda_{\chi \xi} v_{\chi} v_{\xi} & \lambda_{\eta \xi} v_{\eta} v_{\xi} & 2 \lambda_{\xi} v_{\xi}^{2}
\end{array}\right),
$$

which contains a SM-like Higgs boson found by LHC. The mass eigenstates will be discussed using simplified conditions.

Let us summarize the Higgs content:

(1) In the charged scalar sector: there are two Goldstone bosons $\eta^{-}$and $\chi^{-}$eaten by the gauge bosons $W^{-}$ and $Y^{-}$. Three massive charged Higgs bosons are $\phi_{1}^{+}, \phi_{2}^{+}$, and $\phi_{4}^{+}$. The remaining fields $\rho_{1}^{+}, \phi_{3}^{+}$, and $\rho_{3}^{+}$are mixing.

(2) In the $C P$-odd scalar sector: there is one massless Majoron scalar $I_{\xi^{0}}$ which is denoted by $G_{M}$. Fortunately, it is a gauge singlet, therefore, is phenomenologically harmless. Two massless scalars $I_{\eta_{1}^{0}}$ and $I_{\chi_{3}^{0}}$ are Goldstone bosons for the gauge bosons $Z$ and $Z^{\prime}$, respectively. There exists another massless state denoted by $G_{1}$, its role will be discussed below. Here we just mention that in the limit $v_{\eta} \ll v_{\chi}$, this field is $I_{\chi_{1}^{0}}$. The massive $C P$-odd field are $I_{\varphi_{2}}, A_{1}$ and other two $I_{\varphi_{1}}, I_{\rho}$ are mixing.

(3) In the $C P$-even scalar sector: There is one massless field: $R_{G_{2}}$, and in the limit $v_{\eta} \ll v_{\chi}$, it tends to $R_{\chi_{1}^{0}}$.
Combination of $G_{1}$ and $R_{G_{1}}$ is Goldstone boson for neutral bilepton gauge boson $X^{0}$, namely $G_{X^{0}}=$ $\frac{1}{\sqrt{2}}\left(R_{G_{1}}-i G_{1}\right)$. The massive fields are $R_{\varphi_{2}}, H_{1}, H_{2}$ and three massive $R_{\chi}, R_{\eta}, R_{\xi^{0}}$ and the SM-like Higgs boson $h$. Note that there exists degeneracy in Eqs. (50) and (53) when the contribution arising from $Z_{2} \times Z_{4}$ soft breaking scalar interactions is not considered. Thus, the complex scalar $\varphi_{2}$ has mass given by Eq. (50), which is consistent with the prediction in Ref. [19]. To be a DM candidate, the condition (51) can be used to eliminate the terms with large VEVs such as $v_{\chi}$ and $v_{\xi}$. As a result, the mass of the DM candidate is

$$
m_{\varphi_{2}}^{2}=\frac{1}{2} v_{\eta}^{2} \lambda_{2}^{\eta \varphi} .
$$

According [30], the weakly interacting massive particles (WIMP) candidate has mass around $10 \mathrm{GeV}$, implying that $\lambda_{2}^{\eta \varphi} \approx 0.04$. To get the second DM candidate, namely, $\varphi_{1}^{0}$, we have to carefully choose conditions.

Equations (45) and (53) result in a new complex $w$ defined as follows

$$
\omega=\frac{1}{\sqrt{2}}\left(H_{1}-i A_{1}\right), \quad m_{\omega}^{2}=\frac{\lambda_{8} v_{\chi}^{2}}{2 \cos ^{2} \theta_{a}} .
$$

Let us rewrite the Higgs content in terms of the mass eigenstates mentioned above:

$$
\begin{aligned}
\chi & \simeq\left(\begin{array}{c}
G_{X^{0}} \\
G_{Y^{-}} \\
\frac{1}{\sqrt{2}}\left(v_{\chi}+R_{\chi_{3}^{0}}-i G_{Z^{\prime}}\right)
\end{array}\right), \quad \rho=\left(\begin{array}{c}
\rho_{1}^{+} \\
\frac{1}{\sqrt{2}}\left(R_{\rho}-i I_{\rho}\right) \\
\rho_{3}^{+}
\end{array}\right), \quad \eta \simeq\left(\begin{array}{c}
\frac{1}{\sqrt{2}}\left(v_{\eta}+h-i G_{Z}\right) \\
G_{W^{-}} \\
\omega
\end{array}\right), \\
\varphi_{2}^{0} & =\frac{1}{\sqrt{2}}\left(R_{\varphi_{2}}-i I_{\varphi_{2}}\right) \sim(1,1,0, i, 1,0) \sim \mathrm{DM} \text { candidate, } \\
\xi^{0} & =\frac{1}{\sqrt{2}}\left(v_{\xi}+R_{\xi^{0}}-i G_{M}\right) \sim(1,1,0) .
\end{aligned}
$$

\section{A. Simplified solutions}

We have shown that the mass eigenstates of scalars have been determined explicitly, except those relating to the two $3 \times 3$ matrices (38) and (57). By introducing some further constrained assumptions to simplify these matrices so that the physical states can be found, we will point out that the parameter space of the model under consideration contains valid regions, which are consistent with the experiment data including the $\rho$ parameter. To reduce the arbitrary of the unknown Higgs couplings in the potential (A1), the following relations are assumed first

$$
\lambda_{1}=\lambda_{2}, \quad \lambda_{15}=\lambda_{16}, \quad \lambda_{19}=\lambda_{20}, \quad w_{1}=w_{4} .
$$

In the next steps, we just pay attention to find the masses and mass eigenstates of the two matrices (38) and (57). The other will be summarized if necessary.

\section{The CP-odd Higgs bosons}

Under the assumption (61), the $C P$-odd scalar sector consists of four massless fields $\left\{I_{\chi_{3}^{0}}, I_{\eta}, G_{M}, G_{1}\right\}$ and four massive fields $\left\{A_{1}, A_{2}, A_{3} I_{\varphi_{2}}\right\}$, as summarized in Table III. 
TABLE III. Squared mass of $C P$-odd scalars under condition in (61) and $v_{\chi} \gg v_{\eta}$.

\begin{tabular}{lcccccccc}
\hline \hline Fields & $I_{\chi_{1}^{0}}=G_{1} \in G_{X^{0}}$ & $I_{\chi_{3}^{0}}=G_{Z^{\prime}}$ & $I_{\eta_{1}^{0}}=G_{Z}$ & $I_{\eta_{3}^{0}}=A_{1}$ & $I_{\rho}=A_{2}$ & $I_{\varphi_{1}^{0}}=A_{3}$ & $I_{\varphi_{2}^{0}}=D M$ & $I_{\xi^{0}}=G_{M}$ \\
\hline Squared mass & 0 & 0 & 0 & $m_{A_{1}}^{2}$ & $m_{A_{2}}^{2}$ & $m_{A_{3}}^{2}$ & $m_{I_{\varphi_{2}^{0}}}^{2}$ & 0 \\
\hline \hline
\end{tabular}

TABLE IV. Squared masses of $C P$-even scalars under condition in (62) and $v_{\chi} \gg v_{\eta}$.

\begin{tabular}{lcccccccc}
\hline \hline Fields & $R_{\chi_{1}^{0}} \in G_{X^{0}}$ & $R_{\chi_{3}^{0}} \simeq H_{4}$ & $R_{\eta_{1}^{0}}=h$ & $R_{\eta_{3}^{0}}=H_{1}$ & $R_{\rho}=H_{2}$ & $R_{\varphi_{1}^{0}}=H_{3}$ & $R_{\varphi_{2}^{0}}=D M$ & $R_{\xi^{0}} \simeq H_{5}$ \\
\hline Squared mass & 0 & $\lambda v_{\chi}^{2}$ & $\frac{4}{3} \lambda v_{\eta}^{2}$ & $m_{H_{1}}^{2}=m_{A_{1}}^{2}$ & $m_{H_{2}}^{2}$ & $m_{H_{3}}^{2}$ & $m_{R_{\varphi_{2}^{0}}}^{2}=m_{\varphi_{2}^{0}}^{2}$ & $3 \lambda v_{\chi}^{2}$ \\
\hline \hline
\end{tabular}

\section{The CP-even and SM-like Higgs bosons}

Now we turn to the sector where the SM Higgs boson exists, i.e., the matrix in the basis $\left(R_{\chi_{3}^{0}}, R_{\eta_{1}^{0}}, R_{\xi^{0}}\right)$ is given by Eq. (57). Let us assume a simplified scenario worth to be considered is characterized by the following relations:

$\lambda_{5}=\lambda_{13}=\lambda_{17}=\lambda_{\xi}=\lambda_{\chi \xi}=\lambda_{\eta \xi}=\lambda, \quad v_{\xi}=v_{\chi}$.

In this scenario, the squared matrix (57) takes the simple form:

$m_{C \text { even } 3}^{2}=\lambda\left(\begin{array}{rrr}2 x^{2} & x & x \\ x & 2 & 1 \\ x & 1 & 2\end{array}\right) v_{\chi}^{2}, \quad x=\frac{v_{\eta}}{v_{\chi}}=\tan \theta_{a}$.

Because $v_{\chi} \gg v_{\eta}$, the matrix (63) can be perturbatively diagonalized as follows:

$$
\begin{aligned}
R_{C P \text { even } 3}^{T} m_{C P \text { even } 3}^{2} R_{C P \text { even } 3} & \simeq\left(\begin{array}{ccc}
\frac{4}{3} \lambda v_{\eta}^{2} & 0 & 0 \\
0 & \lambda v_{\chi}^{2} & 0 \\
0 & 0 & 3 \lambda v_{\chi}^{2}
\end{array}\right), \\
R_{C P \text { even } 3} & \simeq\left(\begin{array}{ccc}
-1+\frac{x^{2}}{9} & 0 & \frac{\sqrt{2}}{3} x \\
\frac{x}{3} & -\sqrt{\frac{1}{2}} & \sqrt{\frac{1}{2}} \\
\frac{x}{3} & \sqrt{\frac{1}{2}} & \sqrt{\frac{1}{2}}
\end{array}\right),
\end{aligned}
$$

Thus, we find that the physical scalars included in the matrix $m_{C P \text { even3 }}^{2}$ are

$$
\left(\begin{array}{c}
h \\
H_{4} \\
H_{5}
\end{array}\right) \simeq\left(\begin{array}{ccc}
-1+\frac{x^{2}}{9} & \frac{x}{3} & \frac{x}{3} \\
0 & -\sqrt{\frac{1}{2}} & \sqrt{\frac{1}{2}} \\
\frac{\sqrt{2}}{3} x & \sqrt{\frac{1}{2}} & \sqrt{\frac{1}{2}}
\end{array}\right)\left(\begin{array}{l}
R_{\eta_{1}^{0}} \\
R_{\chi_{3}^{0}} \\
R_{\xi^{0}}
\end{array}\right),
$$

where $h$ is the SM-like Higgs boson with mass $126 \mathrm{GeV}$ identified with that found by LHC, whereas $H_{4}$ and $H_{5}$ are physical heavy scalars acquiring masses at the breaking scale of the $S U(3)_{L} \times U(1)_{X} \times Z_{4} \times Z_{2} \times U(1)_{L_{g}}$ symmetry. Thus, we find that $h$ has couplings very close to SM expectation with small deviations of the order of $\frac{v_{n}^{2}}{v_{\chi}^{2}} \sim \mathcal{O}\left(10^{-3}\right)$. In addition, the squared masses of the physical scalars $h$ and $H_{4,5}$ are given in (64).

Now, the content of the $C P$-even scalar sector is summarized in Table IV.

Taking into account mass of the SM Higgs boson equal $126 \mathrm{GeV}$, from Table IV we obtain $\lambda \approx 0.187$, which can be used to calculate masses of the $H_{4,5}$ once $v_{\chi}$ is fixed.

\section{The charged Higgs bosons}

The charged scalar sector contains two massless fields: $G_{W^{+}}$and $G_{Y^{+}}$which are Goldstone bosons eaten by the longitudinal components of the $W^{+}$and $Y^{+}$gauge bosons, respectively. The other massive fields are $\phi_{1}^{+}, \phi_{2}^{+}$, and $\phi_{4}^{+}$ with respective masses given in (39).

In the basis $\left(\rho_{1}^{+}, \rho_{3}^{+}, \phi_{3}^{+}\right)$, the squared mass matrix is given in (38). Let us make effort to simplify this matrix. Note that $\mu_{\chi}^{2}, \mu_{\eta}^{2}$, and $\mu_{\xi}^{2}$ can be derived using relations (35) and (62). In addition, it is reasonable to assume

$$
\mu_{\rho}^{2}=-\frac{v_{\chi}^{2}}{2}\left(\lambda_{18}+\lambda_{\rho \xi}\right) \approx \mu_{\eta}^{2}, \quad \mu_{\phi_{3}^{+}}^{2}=-\frac{v_{\chi}^{2}}{2}\left(\lambda_{2}^{\chi \phi}+\lambda_{2}^{\phi \xi}\right),
$$

we obtain the simple form of the squared mass matrix of the charged Higgs bosons,

$$
M_{\text {chargeds }}^{2}=\left(\begin{array}{ccc}
\frac{1}{2} v_{\eta}^{2}\left(\lambda_{6}+\lambda_{9}\right) & 0 & \frac{\lambda_{3}}{2} v_{\eta} v_{\chi} \\
0 & \frac{1}{2}\left(v_{\chi}^{2} \lambda_{7}+\lambda_{6} v_{\eta}^{2}\right) & \frac{1}{\sqrt{2}} v_{\chi} w_{2} \\
\frac{\lambda_{3}}{2} v_{\eta} v_{\chi} & \frac{1}{\sqrt{2}} v_{\chi} w_{2} & \frac{1}{2} v_{\eta}^{2} \lambda_{2}^{\eta \phi}
\end{array}\right) .
$$

The matrix (67) predicts that there may exist two light charged Higgs bosons $H_{1,2}^{+}$with masses at the electroweak 
TABLE V. Squared mass of charged scalars under condition in (66) and $v_{\chi} \gg v_{\eta}$.

\begin{tabular}{lcccccccc}
\hline \hline Fields & $\eta_{2}^{+}=G_{W^{+}}$ & $\chi_{2}^{+}=G_{Y^{+}}$ & $H_{1}^{+}$ & $H_{3}^{+}$ & $H_{2}^{+}$ & $\phi_{1}^{+}$ & $\phi_{2}^{+}$ & $\phi_{4}^{+}$ \\
\hline Squared mass & 0 & 0 & $m_{H_{1}^{+}}^{2}$ & $m_{H_{3}^{+}}^{2}$ & $m_{H_{2}^{+}}^{2}$ & $m_{\phi_{1}^{+}}^{2}$ & $m_{\phi_{2}^{+}}^{2}$ & $m_{\phi_{4}^{+}}^{2}$ \\
\hline \hline
\end{tabular}

scale and the mass of $H_{3}^{+}$which is mainly composed of $\rho_{3}^{+}$ is around $3.5 \mathrm{TeV}$. In addition, the Higgs boson $H_{1}^{+}$almost does not carry lepton number, whereas the others two do.

Generally, the Higgs potential always contains mass terms which mix VEVs. However, these terms must be small enough to avoid high order divergences (for examples, see Refs. [33,34]) and provide baryon asymmetry of Universe by the strong electroweak phase transition (EWPT).

Ignoring the mixing term containing $\lambda_{3}$ in (67) does not affect other physical aspects, since the above-mentioned term just increases or decreases a small amount of the charged Higgs bosons. Therefore, without loss of generality, neglecting the term with $\lambda_{3}$ satisfies other aims such as EWPT.

Hence, in the matrix of (67), the coefficient $\lambda_{3}$ is reasonably assumed to be zero. Therefore we get immediately one physical field $\rho_{1}^{+}$with mass given by

$$
m_{\rho_{1}^{+}}^{2}=\frac{1}{2} v_{\eta}^{2}\left(\lambda_{6}+\lambda_{9}\right) .
$$

The other fields mix by submatrix given at the bottom of (67). The limit $\rho_{1}^{+}=H_{1}^{+}$when $\lambda_{3}=0$ is very interesting for discussion of the Higgs contribution to the $\rho$ parameter.

The content of the charged scalar sector is summarized in Table V. It is worth mentioning that the masses of three charged scalars $\phi_{i}^{+}, i=1,2,4$ are still not fixed.

The potential including lepton number violations, i.e., $V_{\text {full }}=V_{\mathrm{LNC}}+V_{\mathrm{LNV}}$ is quite similar to the previous one. There are some differences:

(1) The masses of the fields receive some new contributions.

(2) The complex scalar $\varphi_{2}^{0}$ has the same mass in both cases.

(3) Majoron does not exist and its mass only arises from lepton number violating scalar interactions.

(4) The mixing of the $C P$-even scalar fields is more complicated.

\section{B. Scalar contributions to the $\rho$ parameter}

The new Higgs bosons may give contribution to the $\rho$ parameter at one-loop level, as shown in many models beyond the SM, such as the simplified models [35], the two Higgs doublet models [36,37], and the supersymmetric version of the SM [38]. In the 3-3-1 CKS model, we will consider the effect of the Higgs contributions to the $\rho$ parameter at one-loop level. These contributions will be determined in the limit of the suppressed $Z-Z^{\prime}$ mixing and the decoupling of the SM-like Higgs boson with other $C P$-even neutral Higgs bosons. As a consequence, the oneloop contribution of the SM-like Higgs boson to the $\rho$ parameter is the same as in the SM. Excepting for the components of the scalar triplet $\rho$, the other heavy $C P$-even neutral Higgs bosons do not couple with the SM gauge bosons $W$ and $Z$ and thus they do not provide contributions to the $\rho$ parameter. Contributions of the remaining Higgs bosons can be calculated using the results given in Ref. [38]. In particular, contributions of any Higgs bosons in our case to $\Delta \rho$ are determined as follows

$$
\Delta \rho=\frac{\Pi_{W W}(0)}{M_{W}^{2}}-\frac{\Pi_{Z Z}(0)}{M_{Z}^{2}},
$$

where $\Pi_{W W}(0)$ and $\Pi_{Z Z}(0)$ are the coefficients of $-i g_{\mu \nu}$ in the vacuum-polarization amplitudes of charged and neutral $W$ bosons and $Z$ gauge bosons, respectively. Our case relates with only the contribution of "non-Higgs scalars" $\phi_{1,2}$ with masses $m_{1,2}$ and coupling

$i c \phi_{1}^{*} \stackrel{\leftrightarrow}{\partial}_{\mu} \phi_{2} V^{\mu} \equiv i c\left[\phi_{1}^{*} \partial_{\mu} \phi_{2}-\left(\partial_{\mu} \phi_{1}^{*}\right) \phi_{2}\right] V^{\mu}, \quad(V=W, Z)$,

The corresponding contribution is

$$
\Pi(\text { scalar })=\frac{|c|^{2}}{16 \pi^{2}} f_{s}\left(m_{1}, m_{2}\right),
$$

where

$$
\begin{aligned}
f_{s}\left(m_{1}, m_{2}\right)= & f_{s}\left(m_{2}, m_{1}\right)=\frac{m_{1}^{2} m_{2}^{2}}{m_{1}^{2}-m_{2}^{2}} \ln \left[\frac{m_{2}^{2}}{m_{1}^{2}}\right] \\
& +\frac{1}{2}\left(m_{1}^{2}+m_{2}^{2}\right) \\
= & m_{1}^{2} f_{s}(x)=m_{1}^{2}\left(\frac{x \ln (x)}{1-x}+\frac{1+x}{2}\right), \\
x_{21} \equiv & \frac{m_{2}^{2}}{m_{1}^{2}} .
\end{aligned}
$$

The function in Eq. (72) satisfies $f_{s}\left(m_{1}, m_{1}\right)=\lim _{m_{2} \rightarrow m_{1}} \times$ $f_{s}\left(m_{1}, m_{2}\right)=0$ and $f_{s}\left(m_{1}, m_{2}\right)>0$ with $m_{1} \neq m_{2}$. As a consequence, the charged Higgs bosons $\phi_{1,2,4}^{ \pm}$having vanishing Higgs-gauge couplings with other Higgs bosons give vanishing contributions to the $\rho$ parameter. Nonvanishing contributions now may arise from the charged Higgs bosons $H_{1,2,3}^{ \pm}$corresponding to the basis $\left(\rho_{1}^{ \pm}, \rho_{3}^{ \pm}, \phi_{3}^{ \pm}\right)$and the $C P$-odd neutral Higgs relating with $I_{\rho}$. The relevant Lagrangian is 

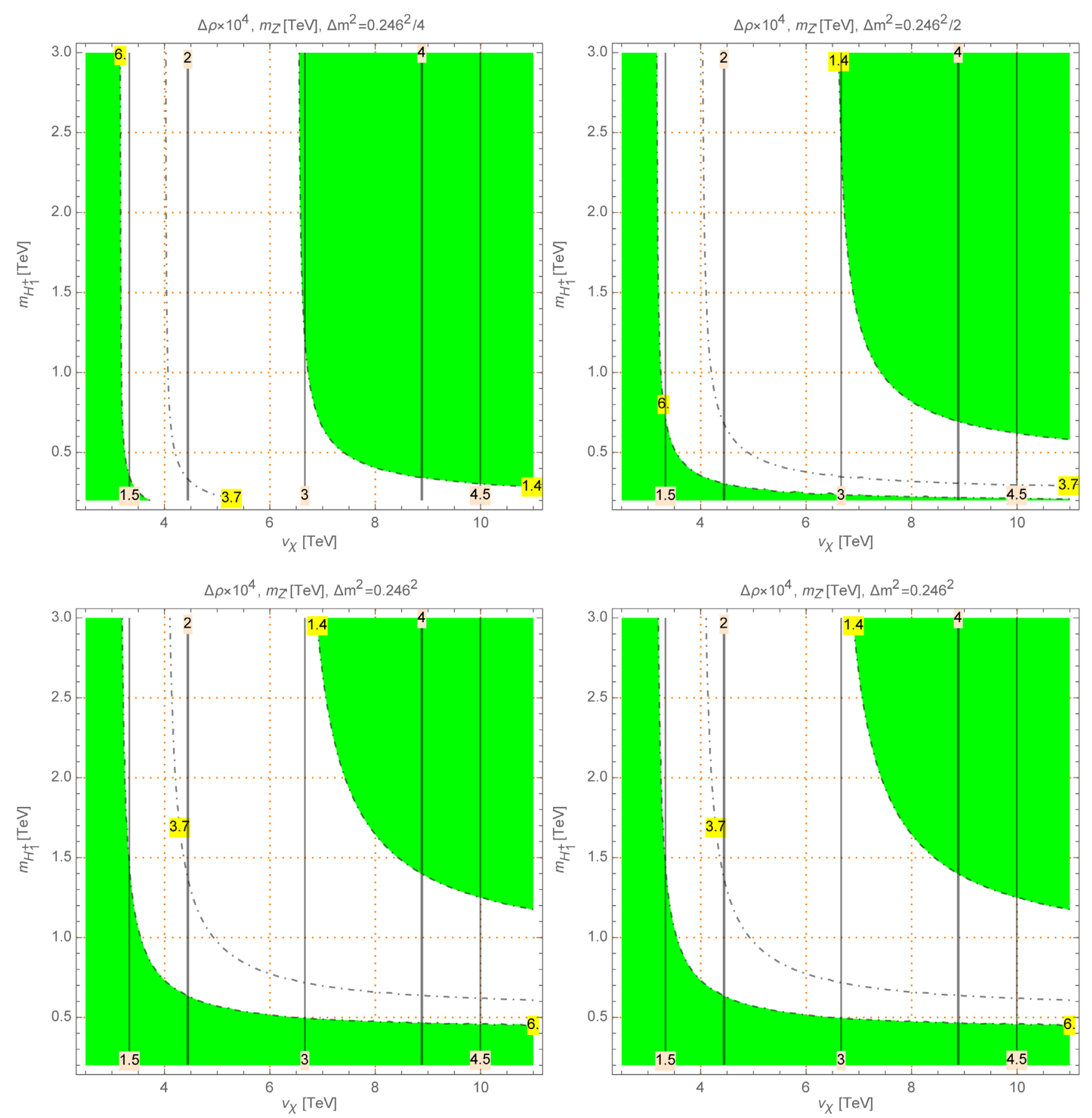

FIG. 2. Contour plots of the $\rho$ parameter (dotted-dashed curves) and $M_{Z^{\prime}}$ (black curves) as functions of $v_{\chi}$ and $m_{H_{1}^{+}}$. The green regions are excluded by the recent experimental constraint of the $\rho$.

$$
\begin{aligned}
\mathcal{L}_{V H H}= & \left(D_{\mu} \rho\right)^{\dagger}\left(D^{\mu} \rho\right)+\left(D_{\mu} \phi_{3}^{+}\right)^{*}\left(D^{\mu} \phi_{3}^{+}\right) \\
\rightarrow & \frac{i g}{2} Z^{\mu}\left[\frac{1-2 s_{W}^{2}}{c_{W}}\left(\rho_{1}^{-} \stackrel{\leftrightarrow}{\partial}_{\mu} \rho_{1}^{+}\right)+\frac{-2 s_{W}^{2}}{c_{W}}\left(\rho_{3}^{-} \stackrel{\leftrightarrow}{\partial}_{\mu} \rho_{3}^{+}\right)+\frac{-i}{c_{W}}\left(I_{\rho} \stackrel{\leftrightarrow}{\partial}_{\mu} R_{\rho}\right)\right] \\
& \left.+\frac{-i g s_{W}^{2}}{c_{W}} Z^{\mu}\left(\phi_{3}^{-} \stackrel{\leftrightarrow}{\partial}_{\mu} \phi_{3}^{+}\right)+\left[-\frac{i g}{2} W^{+\mu} \rho_{1}^{-} \stackrel{\leftrightarrow}{\partial}_{\mu} R_{\rho}-i \stackrel{\leftrightarrow}{\partial}_{\mu} I_{\rho}\right)+\text { H.c. }\right] .
\end{aligned}
$$


In the scalar-gauge interactions of Eq (73), only the last term contributes to $\Pi_{W W}(0)$, thus giving rise to a nonnegative contribution to the $\rho$ parameter, which may make the allowed $M_{Z^{\prime}}$ mass to move outside the excluded region recently reported by LHC searches [39]. On the contrary, all of the remaining terms contributing to $\Pi_{Z Z}(0)$, give nonpositive contributions to the $\rho$ parameter. For illustration, we will consider a simple case where only positive contributions to the $\rho$ parameter are kept, namely $\rho_{1,3}^{ \pm} \equiv$ $H_{1,3}^{ \pm}, \phi_{3}^{ \pm} \equiv H_{2}^{ \pm}, I_{\rho} \equiv A_{2}$ and $R_{\rho}$ are mass eigenstates. Then, all contributions to $\Pi_{Z Z}(0)$ arising from the charged Higgs bosons are proportional to $f_{s}\left(m_{s}, m_{s}\right)=0$ with $s=\rho_{1,3}^{ \pm}$, $\phi_{3}^{ \pm}$. In addition, the simplified condition (61) with $\lambda_{1} \ll 1$ results in $m_{I_{\rho}}^{2}=m_{R_{\rho}}^{2}$, leading to a vanishing neutral Higgs boson contribution to $\Pi_{Z Z}(0): f_{s}\left(m_{I_{\rho}}, m_{R_{\rho}}\right)=0$. The only nonzero contribution has the form

$$
\Delta \rho^{H}=\frac{g^{2}}{16 \pi^{2} m_{W}^{2}} f_{s}\left(m_{H_{1}^{+}}, m_{R_{\rho}}\right)=\frac{\sqrt{2} G_{F}}{16 \pi^{2}} f_{s}\left(m_{H_{1}^{+}}, m_{R_{\rho}}\right),
$$

where

$$
\Delta m^{2} \equiv m_{R_{\rho}}^{2}-m_{H_{1}^{+}}=-\frac{\lambda_{9} v_{\eta}^{2}}{2} \sim \mathcal{O}\left(v_{\eta}^{2}\right) .
$$

Allowed regions of the parameter space for some specific values of $\Delta m^{2}$ are shown in Fig. 2. It can be seen that the large values of $v_{\chi}$ are still allowed, thus implying that no upper bounds are required. The allowed values of $v_{\chi}$ and $M_{Z^{\prime}}$ strongly depend on the lower bound of $m_{H_{1}^{ \pm}}$and $m_{R_{\rho}}$, which may have previously reported from LHC searches. Unfortunately, the Higgs triplets $\rho$ containing the neutral components $\rho_{2}^{0}$ with zero VEV, hence all of the three components of $\rho$ do not couple to the two SM gauge bosons $Z$ and $W$. This Higgs triplet also does not contribute to the SM-like Higgs boson. As a result, all of the Higgs bosons $H_{1}^{ \pm}, R_{\rho}$, and $I_{\rho}$ are not affected by the following decays searched by LHC: $H_{1}^{ \pm} \rightarrow W^{ \pm} Z, W^{ \pm} h$ and $R_{\rho}, I_{\rho} \rightarrow$ $W^{+} W^{-}, Z Z, Z h$. These Higgs bosons do not couple with the SM quarks [19], cannot be produced at LHC from the recent channel searching [40]. Only the allowed tree level decays to two SM fermions are leptonic decays: $H_{1}^{ \pm} \rightarrow$ $\nu_{2,3} \tau, \nu_{2,3} \mu$ and $R_{\rho}, I_{\rho} \rightarrow \bar{e}_{i} e_{i}(i=1,2,3)$, which are also searched by LHC, but the couplings of these Higgs with SM quarks are necessary to produce these Higgs bosons.

Because the above Higgs bosons have couplings with many new charged particles in the model under consideration, their one-loop level decays to photons may appear, thus making them interesting channels for their search at the LHC, in particular these charged Higgs bosons feature the following decay modes $H_{1}^{ \pm} \rightarrow W^{ \pm} \gamma, R_{\rho}, I_{\rho} \rightarrow Z \gamma$ [41], and $R_{\rho}, I_{\rho} \rightarrow \gamma \gamma[42,43]$. The heavy neutral Higgs boson masses are predicted to be at the $\mathrm{TeV}$ scale, which is outside the LHC excluded regions. Combined with the relation (75), the mass of the charged Higgs boson $\mathrm{H}_{1}^{+}$ should also be at the TeV scale. From Fig. 2, we can see that $M_{Z^{\prime}} \geq 4 \mathrm{TeV}$ is allowed if $\Delta m^{2}$ is large enough, for example $\Delta m^{2} \geq(0.246 \mathrm{TeV})^{2}$.

\section{CHARGED LEPTON FLAVOR VIOLATING DECAY CONSTRAINTS}

In this section we will determine the constraints that the charged lepton flavor violating decays $\mu \rightarrow e \gamma, \tau \rightarrow \mu \gamma$ and $\tau \rightarrow e \gamma$ imposed on the parameter space of our model. As mentioned in Ref. [19], the sterile neutrino spectrum of the model is composed of two almost degenerate neutrinos with masses at the Fermi scale and four nearly degenerate neutrinos with $\mathrm{TeV}$ scale masses. These sterile neutrinos together with the heavy $W^{\prime}$ gauge boson induce the $l_{i} \rightarrow l_{j} \gamma$ decay at one loop level, whose branching ratio is given by: [44-46]:

$$
\begin{aligned}
\operatorname{Br}\left(l_{i} \rightarrow l_{j} \gamma\right) & =\frac{\alpha_{W}^{3} s_{W}^{2} m_{l_{i}}^{5}}{256 \pi^{2} m_{W^{\prime}}^{4} \Gamma_{i}}\left|2 G\left(\frac{m_{N_{1}}^{2}}{m_{W^{\prime}}^{2}}\right)+4 G\left(\frac{m_{N_{2}}^{2}}{m_{W^{\prime}}^{2}}\right)\right|^{2} \\
G(x) & =-\frac{2 x^{3}+5 x^{2}-x}{4(1-x)^{2}}-\frac{3 x^{3}}{2(1-x)^{4}} \ln x
\end{aligned}
$$

In our numerical analysis we have fixed $m_{N_{1}}=100 \mathrm{GeV}$ and we have varied the $W^{\prime}$ gauge boson mass in the range $4 \mathrm{TeV} \lesssim m_{W^{\prime}} \lesssim 5 \mathrm{TeV}$. We consider neutral heavy $Z^{\prime}$ gauge boson masses larger than $4 \mathrm{TeV}$ to fulfill the bound arising from the experimental data on $K, D$ and $B$ meson mixings [28]. Figure 3 shows the allowed parameter space in the $m_{W^{\prime}}-m_{N}$ plane consistent with the constraints arising from charged lepton flavor violating decays. As seen from Fig. 3, the obtained values for the branching ratio of $\mu \rightarrow e \gamma$ decay are below its experimental upper limit of $4.2 \times 10^{-13}$ since these values are located in the range $3 \times 10^{-13} \lesssim \operatorname{Br}(\mu \rightarrow e \gamma) \lesssim$ $4 \times 10^{-13}$, for sterile neutrino masses $m_{N_{2}}$ lower than about $1.12 \mathrm{TeV}$. In the same region of parameter space, the obtained branching ratios for the $\tau \rightarrow \mu \gamma$ and $\tau \rightarrow e \gamma$ decays can reach

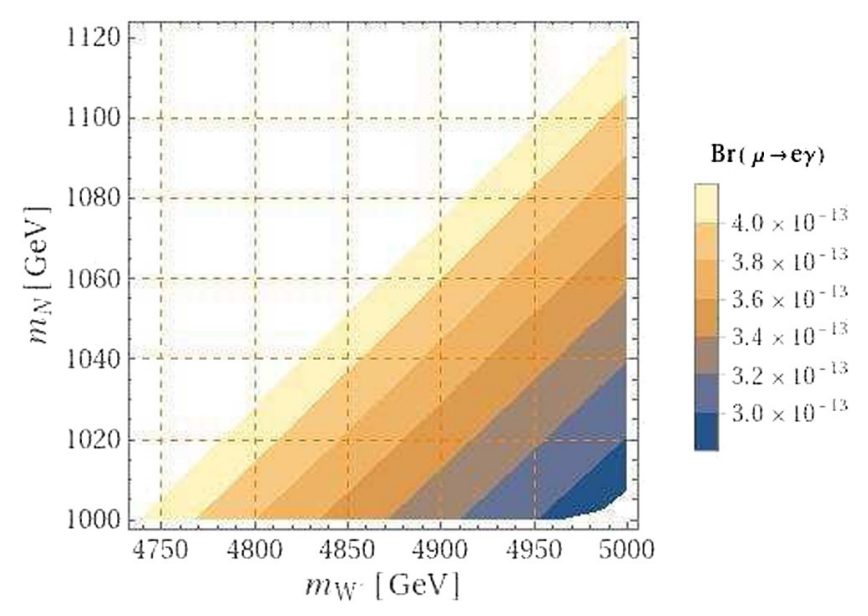

FIG. 3. Allowed parameter space in the $m_{W^{\prime}}-m_{N}$ plane consistent with the LFV constraints. 
values of the order of $10^{-13}$, which is below their corresponding upper experimental bounds of $4.4 \times 10^{-9}$ and $3.3 \times 10^{-9}$, respectively. Consequently, our model is compatible with the charged lepton flavor violating decay constraints provided that the sterile neutrinos are lighter than about $1.12 \mathrm{TeV}$.

\section{SEARCH FOR $Z^{\prime}$ AT LHC}

In this section, we present two typical effects of the LHC, namely, production of single a particle in proton-proton collisions.

\section{A. Phenomenology of $\boldsymbol{Z}^{\prime}$ gauge boson}

In what follows we proceed to compute the total cross section for the production of a heavy $Z^{\prime}$ gauge boson at the LHC via Drell-Yan mechanism. In our computation for the total cross section we consider the dominant contribution due to the parton distribution functions of the light up, down and strange quarks, so that the total cross section for the production of a $Z^{\prime}$ via quark antiquark annihilation in proton proton collisions with center of mass energy $\sqrt{S}$ takes the form:

$$
\begin{aligned}
\sigma_{p p \rightarrow Z^{\prime}}^{(\text {DrellYan })}(S)= & \frac{g^{2} \pi}{6 c_{W}^{2} S}\left\{\left[\left(g_{u L}^{\prime}\right)^{2}+\left(g_{u R}^{\prime}\right)^{2}\right] \int_{\ln \sqrt{\frac{m_{Z^{\prime}}}{S}}}^{-\ln \sqrt{\frac{m_{Z^{\prime}}^{2}}{S}}} f_{p / u}\left(\sqrt{\frac{m_{Z^{\prime}}^{2}}{S}} e^{y}, \mu^{2}\right) f_{p / \bar{u}}\left(\sqrt{\frac{m_{Z^{\prime}}^{2}}{S}} e^{-y}, \mu^{2}\right) d y\right. \\
& +\left[\left(g_{d L}^{\prime}\right)^{2}+\left(g_{d R}^{\prime}\right)^{2}\right] \int_{\ln \sqrt{\frac{m_{Z^{\prime}}}{S}}}^{-\ln \sqrt{\frac{m_{Z^{\prime}}^{2}}{S}}} f_{p / d}\left(\sqrt{\frac{m_{Z^{\prime}}^{2}}{S}} e^{y}, \mu^{2}\right) f_{p / \bar{d}}\left(\sqrt{\frac{m_{Z^{\prime}}^{2}}{S}} e^{-y}, \mu^{2}\right) d y \\
& \left.+\left[\left(g_{d L}^{\prime}\right)^{2}+\left(g_{d R}^{\prime}\right)^{2}\right] \int_{\ln \sqrt{\frac{m_{Z^{\prime}}}{S}}}^{-\ln \sqrt{\frac{m_{Z^{\prime}}^{2}}{S}}} f_{p / s}\left(\sqrt{\frac{m_{Z^{\prime}}^{2}}{S}} e^{y}, \mu^{2}\right) f_{p / \bar{s}}\left(\sqrt{\frac{m_{Z^{\prime}}^{2}}{S}} e^{-y}, \mu^{2}\right) d y\right\}
\end{aligned}
$$

Figure 4 displays the $Z^{\prime}$ total production cross section at the LHC via Drell-Yan mechanism at the LHC for $\sqrt{S}=$ $13 \mathrm{TeV}$ and as a function of the $Z^{\prime}$ mass, which is taken to range from $4 \mathrm{TeV}$ up to $5 \mathrm{TeV}$. We consider neutral heavy $Z^{\prime}$ gauge boson masses larger than $4 \mathrm{TeV}$ to fulfill the bound arising from the experimental data on $K, D$, and $B$ meson mixings [28]. For such as a region of $Z^{\prime}$ masses we find that the total production cross section ranges from $85 \mathrm{fb}$ up to $10 \mathrm{fb}$. The heavy neutral $Z^{\prime}$ gauge boson after being produced it will decay into pair of SM particles, with dominant decay mode into quark-antiquark pairs as shown in detail in Refs. [24,47]. The two body decays of the $Z^{\prime}$ gauge boson in 3-3-1 models have been studied in details in Refs. [47].

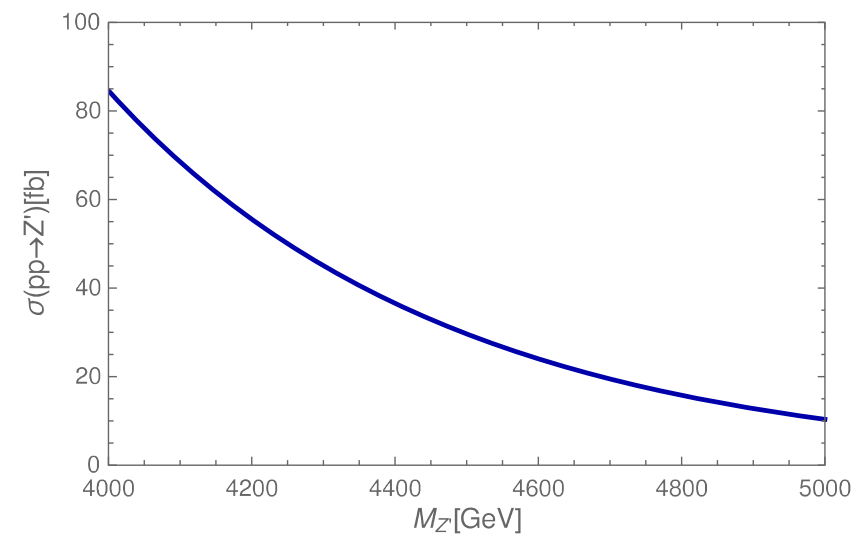

FIG. 4. Total cross section for the $Z^{\prime}$ production via Drell-Yan mechanism at the LHC for $\sqrt{S}=13 \mathrm{TeV}$ and as a function of the $Z^{\prime}$ mass.
In particular, in Ref. [47] it has been shown the $Z^{\prime}$ decays into a lepton pair in 3-3-1 models have branching ratios of the order of $10^{-2}$, which implies that the total LHC cross section for the $p p \rightarrow Z^{\prime} \rightarrow l^{+} l^{-}$resonant production at $\sqrt{S}=$ $13 \mathrm{TeV}$ will be of the order of $1 \mathrm{fb}$ for a $4 \mathrm{TeV} Z^{\prime}$ gauge boson, which is below its corresponding lower experimental limit arising from LHC searches [39]. On the other hand, at the proposed energy upgrade of the LHC at $28 \mathrm{TeV}$ center of mass energy, the total cross section for the Drell-Yan production of a heavy $Z^{\prime}$ neutral gauge boson gets significantly enhanced reaching values ranging from $2.5 \mathrm{pb}$ up to $0.7 \mathrm{pb}$, as indicated in Fig. 5. Consequently, the LHC cross section for the $p p \rightarrow Z^{\prime} \rightarrow l^{+} l^{-}$resonant production at $\sqrt{S}=28 \mathrm{TeV}$

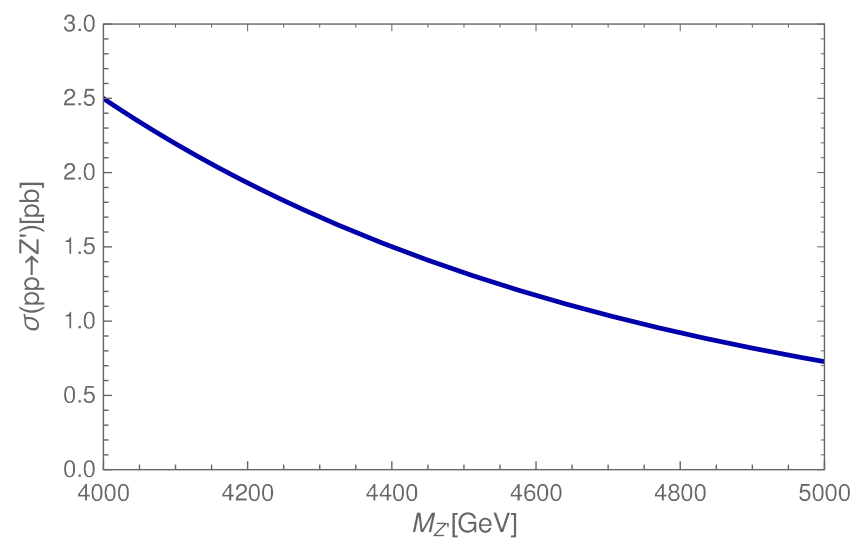

FIG. 5. Total cross section for the $Z^{\prime}$ production via Drell-Yan mechanism at the proposed energy upgrade of the LHC with $\sqrt{S}=28 \mathrm{TeV}$ as a function of the $Z^{\prime}$ mass. 
will be of the order of $10^{-2} \mathrm{pb}$ for a $4 \mathrm{TeV} Z^{\prime}$ gauge boson, which corresponds to the order of magnitude of its corresponding lower experimental limit arising from LHC searches [39].

\section{B. Phenomenology of $\boldsymbol{H}_{\mathbf{4}}$ heavy Higgs boson}

In what follows we proceed to compute the LHC production cross section of the singly heavy scalar $H_{4}$.
Let us note that the singly heavy scalar $H_{4}$ is mainly produced via gluon fusion mechanism mediated by a triangular loop of the heavy exotic quarks $T, J_{1}$, and $J_{2}$. Thus, the total cross section for the production of the heavy scalar $\mathrm{H}_{4}$ through gluon fusion mechanism in proton proton collisions with center of mass energy $\sqrt{S}$ takes the form:

$$
\begin{aligned}
\sigma_{p p \rightarrow g g \rightarrow H_{4}}(S)= & \frac{\alpha_{S}^{2} m_{H_{4}}^{2}\left|\left(R_{C P \text { even } 3}\right)_{22}\right|^{2}}{64 \pi v_{\chi}^{2} S}\left[I\left(\frac{m_{H_{4}}^{2}}{m_{T}^{2}}\right)+I\left(\frac{m_{H_{4}}^{2}}{m_{J_{1}}^{2}}\right)+I\left(\frac{m_{H_{4}}^{2}}{m_{J_{2}}^{2}}\right)\right] \\
& \times \int_{\ln \sqrt{\frac{m_{H_{4}}^{2}}{S}}}^{-\ln \sqrt{\frac{m_{H_{4}}^{2}}{S}}} f_{p / g}\left(\sqrt{\frac{m_{H_{4}}^{2}}{S}} e^{y}, \mu^{2}\right) f_{p / g}\left(\sqrt{\frac{m_{H_{4}}^{2}}{S} e^{-y}}, \mu^{2}\right) d y
\end{aligned}
$$

where $f_{p / g}\left(x_{1}, \mu^{2}\right)$ and $f_{p / g}\left(x_{2}, \mu^{2}\right)$ are the distributions of gluons in the proton which carry momentum fractions $x_{1}$ and $x_{2}$ of the proton, respectively. Furthermore $\mu=m_{H_{4}}$ is the factorization scale and $I(z)$ is given by:

$$
I(z)=\int_{0}^{1} d x \int_{0}^{1-x} d y \frac{1-4 x y}{1-z x y} .
$$

Figure 6 displays the $H_{4}$ total production cross section at the LHC via gluon fusion mechanism for $\sqrt{S}=13 \mathrm{TeV}$, as a function of the $S U(3)_{L} \times U(1)_{X}$ symmetry breaking scale $v_{\chi}$, which is taken to range from $10 \mathrm{TeV}$ up to $20 \mathrm{TeV}$. The aforementioned range of values for the $S U(3)_{L} \times U(1)_{X}$ symmetry breaking scale $v_{\chi}$ corresponds to a heavy scalar mass $m_{H_{4}}$ varying between $4.4 \mathrm{TeV}$ and $8.9 \mathrm{TeV}$. Considering the mass of the heavy scalar field $H_{4}$ in the range $8 \mathrm{TeV} \lesssim M_{H_{4}} \lesssim 8.9 \mathrm{TeV}$, it is reasonable to assume that it will have dominant decay modes into $W^{\prime} W^{\prime}$ and $Z^{\prime} Z^{\prime}$ heavy gauge boson pairs. On the other hand, for a heavy scalar

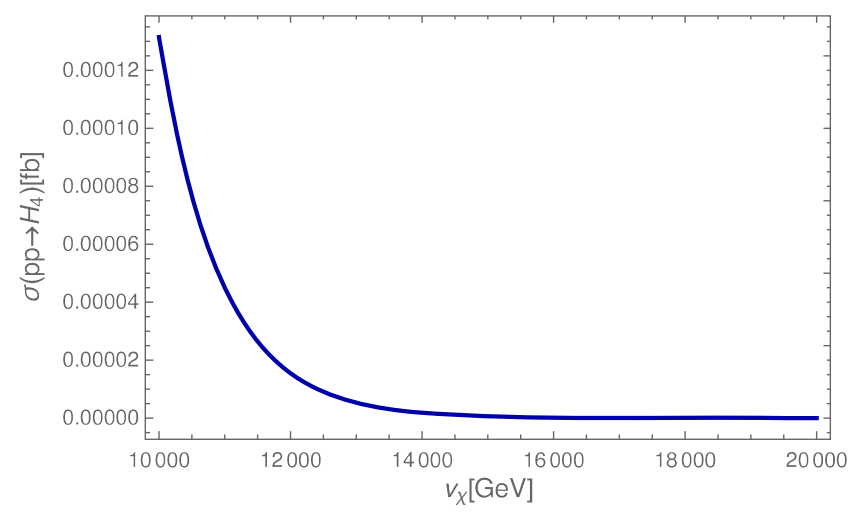

FIG. 6. Total cross section for the $H_{4}$ production via gluon fusion mechanism at the LHC for $\sqrt{S}=13 \mathrm{TeV}$ and as a function of the $S U(3)_{L} \times U(1)_{X}$ symmetry breaking scale $v_{\chi}$ for the simplified scenario described in Eq. (62). field $H_{4}$ with mass in the range $4.4 \mathrm{TeV} \lesssim M_{H_{4}} \lesssim 8 \mathrm{TeV}$, based on Ref. [48], it is reasonable to assume that its dominant decay mode will be on $t \bar{t}$ pair. Furthermore, in the region of $\mathrm{H}_{4}$ masses considered in our analysis, the $\mathrm{H}_{4}$ decay into exotic quark pairs will be kinematically forbidden for exotic quark Yukawa couplings of order unity. Note that we have chosen values for $v_{\chi}$ larger than $10 \mathrm{TeV}$, which corresponds to a $Z^{\prime}$ gauge boson heavier than $4 \mathrm{TeV}$, which is required to guarantee the consistency of 331 models with the experimental data on $K, D$, and $B$ meson mixings [28]. Here, for the sake of simplicity we have restricted to the simplified scenario described by Eq. (62) and we have chosen the exotic quark Yukawa couplings equal to unity, i.e., $y^{(T)}=y^{\left(J_{1}\right)}=y^{\left(J_{2}\right)}=1$. In addition, the top quark mass has been taken to be equal to $m_{t}=173 \mathrm{GeV}$. We find that the total cross section for the production of the $H_{4}$ scalar at the LHC takes a value close to about $10^{-4} \mathrm{fb}$ for the lower bound of $10 \mathrm{TeV}$ of the

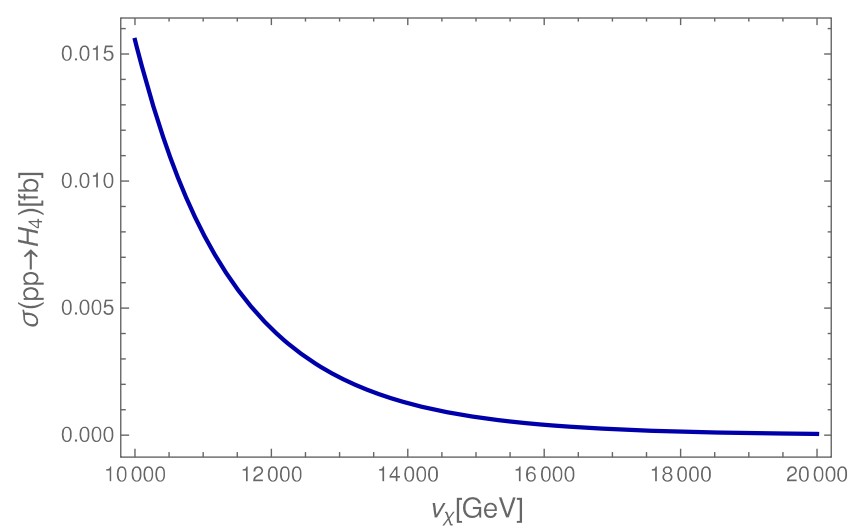

FIG. 7. Total cross section for the $H_{4}$ production via gluon fusion mechanism at the proposed energy upgrade of the LHC with $\sqrt{S}=28 \mathrm{TeV}$ as a function of the $S U(3)_{L} \times U(1)_{X}$ symmetry breaking scale $v_{\chi}$ for the simplified scenario described in Eq. (62). 
$S U(3)_{L} \times U(1)_{X}$ symmetry breaking scale $v_{\chi}$ arising from the experimental data on $K, D$, and $B$ meson mixings [28] and decreases when $v_{\chi}$ takes larger values. We see that the total cross section at the LHC for the $H_{4}$ production via gluon fusion mechanism is small to give rise to a signal for the allowed values of the $S U(3)_{L} \times U(1)_{X}$ symmetry breaking scale $v_{\chi}$. A similar situation happens at the proposed energy upgrade of the LHC with $\sqrt{S}=28 \mathrm{TeV}$, where this total cross section takes a value of $1.6 \times 10^{-2} \mathrm{fb}$ for $v_{\chi}=10 \mathrm{TeV}$ as shown in Fig. 7. Because of the very small $\mathrm{H}_{4}$ production cross section, we do not perform a detailed study of its decay modes. It is worth mentioning that the smoking gun signatures of the model under consideration will be the $Z^{\prime}$ production and the charged lepton flavor violating decay $\mu \rightarrow e \gamma$, whose observation will be crucial to assess to viability of this model.

\section{DARK MATTER RELIC DENSITY}

In this section we provide a discussion of the implications of our model for DM, assuming that the DM candidate is a scalar. Let us recall that our goal in this section is to provide an estimate of the DM relic density in our model, under some simplifying assumptions motivated by the large number of scalar fields of the model. We do not intend to provide a sophisticated analysis of the DM constraints of the model under consideration, which is beyond the scope of the present paper. We just intend to show that our model can accommodate the observed value of the DM relic density, by having a scalar DM candidate with a mass in the $\mathrm{TeV}$ range and a quartic scalar coupling of the order unity, within the perturbative regime. We start by surveying the possible scalar DM candidates in the model. Considering that the $Z_{4}$ symmetry is preserved and taking into account the scalar assignments under this symmetry, given by Eq. (1), we can assign this role to either any of the $S U(3)_{L}$ scalar singlets, i.e., $\operatorname{Re} \varphi_{n}^{0}$ and $\operatorname{Im} \varphi_{n}^{0}(n=1,2)$. In this work we assume that the $\varphi_{I}=\operatorname{Im} \varphi_{1}^{0}$ is the lightest among the $\operatorname{Re} \varphi_{n}^{0}$ and $\operatorname{Im} \varphi_{n}^{0}(n=1,2)$ scalar fields and also lighter than the exotic charged fermions, as well as lighter than $\Psi_{R}$, thus implying that its tree-level decays are kinematically forbidden. Consequently, in this mass range the $\operatorname{Im} \varphi_{1}^{0}$ scalar field is stable.

The relic density is given by (cf. Ref. [30,49])

$$
\Omega h^{2}=\frac{0.1 p b}{\langle\sigma v\rangle}, \quad\langle\sigma v\rangle=\frac{A}{n_{\mathrm{eq}}^{2}}=\frac{\frac{T}{32 \pi^{4}} \int_{4 m_{\varphi}^{2}}^{\infty} \sum_{p=W, Z, t, b, h} g_{p}^{2} \frac{s \sqrt{s-4 m_{\varphi}^{2}}}{2} v_{\mathrm{rel}} \sigma(\varphi \varphi \rightarrow p \bar{p}) K_{1}\left(\frac{\sqrt{s}}{T}\right) d s}{\left(\frac{T}{2 \pi^{2}} \sum_{p=W, Z, t, b, h} g_{p} m_{\varphi}^{2} K_{2}\left(\frac{m_{\varphi}}{T}\right)\right)^{2}},
$$

where $\langle\sigma v\rangle$ is the thermally averaged annihilation cross section, $A$ is the total annihilation rate per unit volume at temperature $T$, and $n_{\mathrm{eq}}$ is the equilibrium value of the particle density. Furthermore, $K_{1}$ and $K_{2}$ are modified Bessel functions of the second kind and order 1 and 2, respectively [49] and $m_{\varphi}=m_{\operatorname{Im} \varphi}$. Let us note that we assume that our scalar DM candidate is a stable weakly interacting particle (WIMP) with annihilation cross sections mediated by electroweak interactions mainly through the Higgs field. In addition we assume that the decoupling of the nonrelativistic WIMP of our model is supposed to happen at a very low temperature. Because of this reason, for the computation of the relic density, we take $T=m_{\varphi} / 20$ as in Ref. [49], corresponding to a typical freeze-out temperature. We assume that our DM candidate $\varphi$ annihilates mainly into $W W, Z Z, t \bar{t}, b \bar{b}$, and $h h$, with annihilation cross sections given by the following relations [50]:

$$
\begin{aligned}
v_{\text {rel }} \sigma\left(\varphi_{I} \varphi_{I} \rightarrow W W\right) & =\frac{\lambda_{h^{2} \varphi^{2}}^{2}}{8 \pi} \frac{s\left(1+\frac{12 m_{W}^{4}}{s^{2}}-\frac{4 m_{W}^{2}}{s}\right)}{\left(s-m_{h}^{2}\right)^{2}+m_{h}^{2} \Gamma_{h}^{2}} \sqrt{1-\frac{4 m_{W}^{2}}{s}} \\
v_{\text {rel }} \sigma\left(\varphi_{I} \varphi_{I} \rightarrow Z Z\right) & =\frac{\lambda_{h^{2} \varphi^{2}}^{2}}{16 \pi} \frac{s\left(1+\frac{12 m_{Z}^{4}}{s^{2}}-\frac{4 m_{Z}^{2}}{s}\right)}{\left(s-m_{h}^{2}\right)^{2}+m_{h}^{2} \Gamma_{h}^{2}} \sqrt{1-\frac{4 m_{Z}^{2}}{s}} \\
v_{\text {rel }} \sigma\left(\varphi_{I} \varphi_{I} \rightarrow q \bar{q}\right) & =\frac{N_{c^{2}} \lambda_{h^{2} \varphi^{2}}^{2} m_{q}^{2}}{4 \pi} \frac{\sqrt{\left(1-\frac{4 m_{f}^{2}}{s}\right)^{3}}}{\left(s-m_{h}^{2}\right)^{2}+m_{h}^{2} \Gamma_{h}^{2}}, \\
v_{\text {rel }} \sigma\left(\varphi_{I} \varphi_{I} \rightarrow h h\right) & =\frac{\lambda_{h^{2} \varphi^{2}}^{2}}{16 \pi s}\left(1+\frac{3 m_{h}^{2}}{s-m_{h}^{2}}-\frac{4 \lambda_{h^{2} \varphi^{2}} v^{2}}{s-2 m_{h}^{2}}\right)^{2} \sqrt{1-\frac{4 m_{h}^{2}}{s}}
\end{aligned}
$$




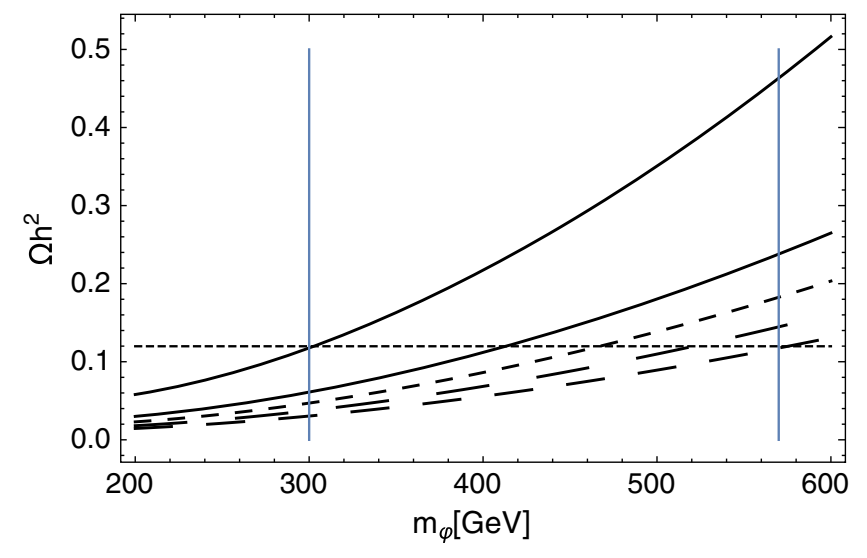

FIG. 8. Relic density $\Omega h^{2}$, as a function of the mass $m_{\varphi}$ of the $\varphi$ scalar field, for several values of the quartic scalar coupling $\lambda_{h^{2} \varphi^{2}}$. The curves from top to bottom correspond to $\lambda_{h^{2} \varphi^{2}}=0.5,0.7$, $0.8,0.9,1$, respectively. The horizontal line shows the observed value $\Omega h^{2}=0.1198$ [54] for the relic density. The vertical lines correspond to the obtained lower and upper limits $300 \mathrm{GeV}$ and $570 \mathrm{GeV}$, respectively, of the mass $m_{\varphi}$ of the scalar dark matter candidate consistent with the experimental measurement of the dark matter relic density.

where $\sqrt{s}$ is the center-of-mass energy, $N_{c}=3$ is the color factor, $m_{h}=125.7 \mathrm{GeV}$ and $\Gamma_{h}=4.1 \mathrm{MeV}$ are the SM Higgs boson $h$ mass and its total decay width, respectively. Note that we have worked on the decoupling limit where the couplings of the $126 \mathrm{GeV}$ Higgs boson to SM particles and its self-couplings correspond to the SM expectation.

The vacuum stability and tree level unitarity constraints of the scalar potential are [51-53]

$$
\begin{gathered}
\lambda_{h^{4}}>0, \quad \lambda_{\varphi^{4}}>0, \quad \lambda_{h^{2} \varphi^{2}}^{2}<\frac{2}{3} \lambda_{h^{4}} \lambda_{\varphi^{4}} . \\
\lambda_{\varphi^{4}}<8 \pi, \quad \lambda_{h^{2} \varphi^{2}}<4 \pi .
\end{gathered}
$$

The dark matter relic density as a function of the mass $m_{\varphi}$ of the scalar field $\varphi_{I}$ is shown in Fig. 8, for several values of the quartic scalar coupling $\lambda_{h^{2} \varphi^{2}}^{2}$, set to be equal to $0.7,0.8$ and 0.9 (from top to bottom). The horizontal line corresponds to the experimental value $\Omega h^{2}=0.1198$ for the relic density. We found that the DM relic density constraint gives rise to a linear correlation between the quartic scalar coupling $\lambda_{h^{2} \varphi^{2}}$ and the mass $m_{\varphi}$ of the scalar DM candidate $\varphi_{I}$, as indicated in Fig. 9.

We find that we can reproduce the experimental value $\Omega h^{2}=0.1198 \pm 0.0026$ [54] of the DM relic density, when the mass $m_{\varphi}$ of the scalar field $\varphi_{I}$ is in the range $300 \mathrm{GeV} \lesssim m_{\varphi} \lesssim 570 \mathrm{GeV}$, for a quartic scalar coupling $\lambda_{h^{2} \varphi^{2}}$ in the window $0.5 \lesssim \lambda_{h^{2} \varphi^{2}} \lesssim 1$, which is consistent with the vacuum stability and unitarity constraints shown in Eqs. (80) and (81). Note that our range of values chosen for the quartic scalar coupling $\lambda_{h^{2}} \varphi^{2}$ also allow

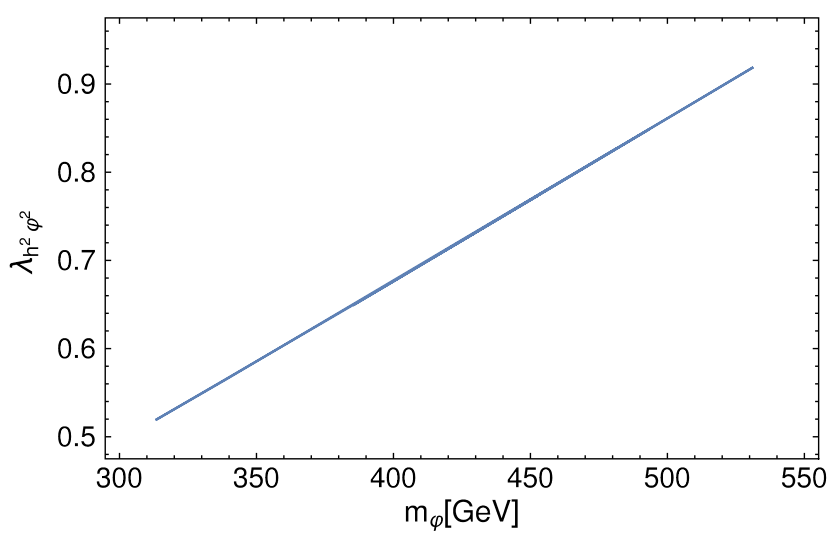

FIG. 9. Correlation between the quartic scalar coupling and the mass $m_{\varphi}$ of the scalar DM candidate $\varphi$, consistent with the experimental value $\Omega h^{2}=0.1198$ for the relic density.

the extrapolation of our model at high energy scales as well as the preservation of perturbativity at one loop level.

\section{CONCLUSIONS}

We have studied some phenomenological aspects of the extended inert 331 model, which incorporates the mechanism of sequential loop-generation of the SM fermion masses, explaining the observed strong hierarchies between them as well as the corresponding mixing parameters. A particular emphasis has been made on analyzing the constraints arising from the experimental data on the $\rho$ parameter, as well as those ones resulting from the charged lepton flavor violating process $\mu \rightarrow e \gamma$ and dark matter. Furthermore, we have studied the production of the heavy $Z^{\prime}$ gauge boson in proton-proton collisions via the DrellYan mechanism. We found that the corresponding total cross section at the LHC ranges from $85 \mathrm{fb}$ up to $10 \mathrm{fb}$ when the $Z^{\prime}$ gauge boson mass is varied within a $4-5 \mathrm{TeV}$ interval. The $Z^{\prime}$ production cross section gets significantly enhanced at the proposed energy upgrade of the LHC with $\sqrt{S}=28 \mathrm{TeV}$ reaching the typical values of $2.5-0.7 \mathrm{pb}$. From these results we find that the $p p \rightarrow Z^{\prime} \rightarrow l^{+} l^{-}$ resonant production cross section reach values of about $1 \mathrm{fb}$ and $10^{-2} \mathrm{pb}$ at $M_{Z^{\prime}}=4 \mathrm{TeV}$, for $\sqrt{S}=14 \mathrm{TeV}$ and $\sqrt{S}=28 \mathrm{TeV}$, respectively. These obtained values for the $p p \rightarrow Z^{\prime} \rightarrow l^{+} l^{-}$resonant production cross sections are below and of the same order of magnitude of its corresponding lower experimental limit arising from LHC searches, for $\sqrt{S}=13 \mathrm{TeV}$ and $\sqrt{S}=28 \mathrm{TeV}$, respectively. Besides that, we have found that $Z^{\prime}$ gauge bosons heavier than about $4 \mathrm{TeV}$ comply with the experimental constraints on the oblique $\rho$ parameter as well as with the collider constraints. In addition, we have found that the constraint on the charged lepton flavor violating decay $\mu \rightarrow e \gamma$ set the sterile neutrino masses to be lighter than about $1.12 \mathrm{TeV}$. We have found that the obtained values of the branching ratio for the $\mu \rightarrow e \gamma$ decay are located in the 
range $3 \times 10^{-13} \lesssim \operatorname{Br}(\mu \rightarrow e \gamma) \lesssim 4 \times 10^{-13}$, whereas the obtained branching ratios for the $\tau \rightarrow \mu \gamma$ and $\tau \rightarrow e \gamma$ decays can reach values of the order of $10^{-13}$. Consequently, our model predicts charged lepton flavor violating decays within the reach of future experimental sensitivity. We found that the total cross section for the production of the $H_{4}$ scalar at the LHC with $\sqrt{S}=13 \mathrm{TeV}$ takes a value close to about $10^{-4} \mathrm{fb}$ for the lower bound of $10 \mathrm{TeV}$ of the $S U(3)_{L} \times U(1)_{X}$ symmetry breaking scale $v_{\chi}$ required by the consistency of the $\rho$ parameter with the experimental data. This value is increased to $1.6 \times 10^{-2} \mathrm{fb}$ at the proposed energy upgrade of the LHC with $\sqrt{S}=28 \mathrm{TeV}$, thus implying that the total cross section at the LHC for the $H_{4}$ production via gluon fusion mechanism is very small to give rise to a signal for the allowed values of the $S U(3)_{L} \times$ $U(1)_{X}$ symmetry breaking scale $v_{\chi}$ even at the proposed energy upgrade of the LHC. We also analyzed in detail the scalar potential and the gauge sector of the model.

The general Higgs sector is separated into two parts. The first part consists of lepton number conserving terms and the second one contains lepton number violating couplings. The first part of potential was considered in details and the SM Higgs boson was derived and as expected, mainly arises from $\eta_{1}^{0}$. We have shown that the whole scalar potential, excepting its $C P$-even sector, has a quite similar situation since the resulting physical scalar mass spectrum is similar in both cases. The scalar spectrum contains enough number of Goldstone bosons for massive gauge bosons. In the $C P$-odd scalar sector, there are four massive bosons and one of them is a DM candidate. The $C P$-even scalar mass spectrum consists of seven massive fields including the SM Higgs boson and a DM candidate. The singly electrically charged Higgs boson sector contains six massive fields. Two of them have masses at the electroweak scale and the remaining one has a mass around $3.5 \mathrm{TeV}$. The masses for the three charged bosons $\phi_{i}^{+}, i=1,2,4$ are not fixed. The scalar potential contains a Majoron but it is harmless, because it is a scalar singlet. Due to the unbroken $Z_{4}$ symmetry our model has the stable scalar dark matter candidates $\operatorname{Re} \varphi_{n}^{0}$ and $\operatorname{Im} \varphi_{n}^{0}(n=1,2)$ and the fermionic dark matter candidate $\Psi_{R}$. In this work we assume that $\varphi_{I}=\operatorname{Im} \varphi_{1}^{0}$ is the lightest among the $\operatorname{Re} \varphi_{n}^{0}$ and $\operatorname{Im} \varphi_{n}^{0}$ $(n=1,2)$ scalar fields and also lighter than the exotic charged fermions and than $\Psi_{R}$, which implies that it is stable and thus it is the dark matter candidate considered in this work. To reproduce the dark matter relic density, the mass of the scalar dark matter candidate has to be in the range $300 \mathrm{GeV} \lesssim m_{\varphi} \lesssim 570 \mathrm{GeV}$, for a quartic scalar coupling $\lambda_{h^{2} \varphi^{2}}$ in the window $0.5 \lesssim \lambda_{h^{2} \varphi^{2}} \lesssim 1$. In addition, it has been shown in Ref. [19] that requiring that the DM candidate $\varphi^{0}$ lifetime be greater than the universe lifetime $\tau_{u} \approx 13.8 \mathrm{Gyr}$ and assuming $m_{\varphi^{0}} \sim 1 \mathrm{TeV}$, we estimate the cutoff scale of our model $\Lambda>3 \times 10^{10} \mathrm{GeV}$. Thus we conclude that under the above specified conditions the model contains viable fermionic $\Psi_{R}$ and scalar $\varphi^{0} \mathrm{DM}$ candidates. A sophisticated analysis of the DM constraints of our model is beyond the scope of the present paper and is left for future studies.

\section{ACKNOWLEDGMENTS}

This research has been financially supported by Fondecyt (Chile), Grant No. 1170803, CONICYT PIA/Basal FB0821, the Vietnam National Foundation for Science and Technology Development (NAFOSTED) under Grant No. 103.012017.356. H. N.L. is very grateful to the Bogoliubov Laboratory for Theoretical Physics, JINR, Dubna, Russia for the warm hospitality during his visit.

\section{APPENDIX: THE SCALAR POTENTIAL}

The renormalizable potential contain three parts: the first one invariant under group $\mathcal{G}$ in (1) is given by

$$
\begin{aligned}
V_{\mathrm{LNC}}= & \mu_{\chi}^{2} \chi^{\dagger} \chi+\mu_{\rho}^{2} \rho^{\dagger} \rho+\mu_{\eta}^{2} \eta^{\dagger} \eta+\sum_{i=1}^{4} \mu_{\phi_{i}^{+}}^{2} \phi_{i}^{+} \phi_{i}^{-}+\sum_{i=1}^{2} \mu_{\varphi_{i}}^{2} \varphi_{i}^{0} \varphi_{i}^{0 *}+\mu_{\xi}^{2} \xi^{0 *} \xi^{0} \\
& +\chi^{\dagger} \chi\left(\lambda_{13} \chi^{\dagger} \chi+\lambda_{18} \rho^{\dagger} \rho+\lambda_{5} \eta^{\dagger} \eta\right)+\rho^{\dagger} \rho\left(\lambda_{14} \rho^{\dagger} \rho+\lambda_{6} \eta^{\dagger} \eta\right)+\lambda_{17}\left(\eta^{\dagger} \eta\right)^{2} \\
& +\lambda_{7}\left(\chi^{\dagger} \rho\right)\left(\rho^{\dagger} \chi\right)+\lambda_{8}\left(\chi^{\dagger} \eta\right)\left(\eta^{\dagger} \chi\right)+\lambda_{9}\left(\rho^{\dagger} \eta\right)\left(\eta^{\dagger} \rho\right) \\
& +\chi^{\dagger} \chi\left(\sum_{i=1}^{4} \lambda_{i}^{\chi \phi} \phi_{i}^{+} \phi_{i}^{-}+\sum_{i=1}^{2} \lambda_{i}^{\chi \varphi} \varphi_{i}^{0} \varphi_{i}^{0 *}+\lambda_{\chi^{\xi}} \xi^{0 *} \xi^{0}\right) \\
& +\rho^{\dagger} \rho\left(\sum_{i=1}^{4} \lambda_{i}^{\rho \phi} \phi_{i}^{+} \phi_{i}^{-}+\sum_{i=1}^{2} \lambda_{i}^{\rho \varphi} \varphi_{i}^{0} \varphi_{i}^{0 *}+\lambda_{\rho_{\xi} \xi^{0 *} \xi^{0}}\right) \\
& +\eta^{\dagger} \eta\left(\sum_{i=1}^{4} \lambda_{i}^{\eta \phi} \phi_{i}^{+} \phi_{i}^{-}+\sum_{i=1}^{2} \lambda_{i}^{\eta \varphi} \varphi_{i}^{0} \varphi_{i}^{0 *}+\lambda_{\eta \xi} \xi^{0 *} \xi^{0}\right)
\end{aligned}
$$




$$
\begin{aligned}
& +\sum_{i=1}^{4} \phi_{i}^{+} \phi_{i}^{-}\left(\sum_{j=1}^{4} \lambda_{i j}^{\phi \phi} \phi_{j}^{+} \phi_{j}^{-}+\sum_{j=1}^{2} \lambda_{i j}^{\phi \varphi} \varphi_{j}^{0} \varphi_{j}^{0 *}+\lambda_{i}^{\phi \xi} \xi^{0 *} \xi^{0}\right) \\
& +\sum_{i=1}^{2} \varphi_{i}^{0} \varphi_{i}^{0 *}\left(\sum_{j=1}^{2} \lambda_{i j}^{\varphi \varphi} \varphi_{j}^{0} \varphi_{j}^{0 *}+\lambda_{i}^{\varphi \xi} \xi^{0 *} \xi^{0}\right)+\lambda_{\xi}\left(\xi^{0 *} \xi^{0}\right)^{2} \\
& +\left\{\lambda_{10}\left(\phi_{2}^{+}\right)^{2}\left(\phi_{3}^{-}\right)^{2}+\lambda_{11}\left(\phi_{2}^{+}\right)^{2}\left(\phi_{4}^{-}\right)^{2}+\lambda_{12}\left(\phi_{3}^{+}\right)^{2}\left(\phi_{4}^{-}\right)^{2}+w_{1}\left(\varphi_{2}^{0}\right)^{2} \varphi_{1}^{0}+w_{2} \chi^{\dagger} \rho \phi_{3}^{-}+w_{3} \eta^{\dagger} \chi \xi^{0}\right. \\
& +w_{4}\left(\varphi_{2}^{0}\right)^{2} \varphi_{1}^{0 *}+w_{5} \phi_{3}^{+} \phi_{4}^{-} \varphi_{1}^{0}+w_{6} \phi_{3}^{+} \phi_{4}^{-} \varphi_{1}^{0 *}+\chi \rho \eta\left(\lambda_{1} \varphi_{1}^{0}+\lambda_{2} \varphi_{1}^{0 *}\right)+\chi^{\dagger} \rho \phi_{4}^{-}\left(\lambda_{15} \varphi_{1}^{0}+\lambda_{16} \varphi_{1}^{0 *}\right) \\
& +\lambda_{3} \eta^{\dagger} \rho \phi_{3}^{-} \xi^{0}+\lambda_{4} \phi_{1}^{+} \phi_{2}^{-} \varphi_{2}^{0} \xi^{0}+\left(\lambda_{19} \phi_{3}^{-} \phi_{4}^{+}+\lambda_{20} \phi_{3}^{+} \phi_{4}^{-}\right)\left(\varphi_{2}^{0}\right)^{2}+\lambda_{21}\left(\varphi_{1}^{0}\right)^{3} \varphi_{1}^{0 *} \\
& \left.+\left(\lambda_{22} \chi^{\dagger} \chi+\lambda_{23} \rho^{\dagger} \rho+\lambda_{24} \eta^{\dagger} \eta+\sum_{i=1}^{4} \lambda_{61 i} \phi_{i}^{+} \phi_{i}^{-}+\sum_{i=1}^{2} \lambda_{62 i} \varphi_{i}^{0} \varphi_{i}^{0 *}+\lambda_{25} \xi^{0 *} \xi^{0}\right)\left(\varphi_{1}^{0}\right)^{2}+\mathrm{H} . \mathrm{c} .\right\} .
\end{aligned}
$$

The second part is a lepton number violating one (the subgroup $U(1)_{L_{g}}$ is violated)

$$
\begin{aligned}
V_{\mathrm{LNV}}= & \mu_{\chi \eta}^{2}\left(\chi^{\dagger} \eta+\eta^{\dagger} \chi\right)+\left[\lambda_{26}\left(\chi^{\dagger} \chi\right)+\lambda_{27}\left(\rho^{\dagger} \rho\right)+\lambda_{28}\left(\eta^{\dagger} \eta\right)\right]\left(\chi^{\dagger} \eta+\eta^{\dagger} \chi\right) \\
& +\lambda_{29}\left[\left(\chi^{\dagger} \eta\right)^{2}+\left(\eta^{\dagger} \chi\right)^{2}\right]+\lambda_{30}\left[\left(\eta^{\dagger} \rho\right)\left(\rho^{\dagger} \chi\right)+\left(\chi^{\dagger} \rho\right)\left(\rho^{\dagger} \eta\right)\right] \\
& +\left\{\xi^{0}\left(w_{7} \chi^{\dagger} \chi+w_{8} \rho^{\dagger} \rho+w_{9} \eta^{\dagger} \eta+\sum_{i=1}^{4} w_{2 i} \phi_{i}^{+} \phi_{i}^{-}+\sum_{i=1}^{2} w_{3 i} \varphi_{i}^{0} \varphi_{i}^{0 *}+w_{10} \xi^{0 *} \xi^{0}\right)\right. \\
& +\xi^{0}\left[w_{11} \chi^{\dagger} \eta+w_{12}\left(\varphi_{1}^{0}\right)^{2}+w_{13}\left(\varphi_{1}^{0 *}\right)^{2}+w_{14}\left(\xi^{0}\right)^{2}\right]+w_{15} \eta^{\dagger} \rho \phi_{3}^{-}+w_{16} \phi_{2}^{-} \phi_{1}^{+} \varphi_{2}^{0} \\
& +\left(\xi^{0}\right)^{2}\left[\lambda_{31} \chi^{\dagger} \chi+\lambda_{32} \rho^{\dagger} \rho+\lambda_{33} \eta^{\dagger} \eta+\sum_{i=1}^{4} \lambda_{63 i} \phi_{i}^{+} \phi_{i}^{-}+\sum_{i=1}^{2} \lambda_{64 i} \varphi_{i}^{0} \varphi_{i}^{0 *}+\lambda_{34} \xi^{0 *} \xi^{0}+\lambda_{35}\left(\varphi_{1}^{0}\right)^{2}+\lambda_{36}\left(\varphi_{1}^{0 *}\right)^{2}\right] \\
& +\chi^{\dagger} \eta\left[\sum_{i=1}^{4} \lambda_{65 i} \phi_{i}^{+} \phi_{i}^{-}+\sum_{i=1}^{2} \lambda_{66 i} \varphi_{i}^{0} \varphi_{i}^{0 *}+\lambda_{37} \xi^{0 *} \xi^{0}+\lambda_{38}\left(\varphi_{1}^{0}\right)^{2}+\lambda_{39}\left(\varphi_{1}^{0 *}\right)^{2}+\lambda_{40}\left(\xi^{0}\right)^{2}+\lambda_{41}\left(\xi^{0 *}\right)^{2}\right] \\
& +\eta^{\dagger} \rho\left(\lambda_{42} \phi_{4}^{-} \varphi_{1}^{0}+\lambda_{43} \phi_{4}^{-} \varphi_{1}^{0 *}+\lambda_{44} \phi_{3}^{-} \xi^{0 *}\right)+\rho^{\dagger} \chi \phi_{3}^{+}\left(\lambda_{45} \xi^{0}+\lambda_{46} \xi^{0 *}\right) \\
& +\lambda_{47}\left(\phi_{1}^{+}\right)^{2} \phi_{3}^{-} \phi_{4}^{-}+\phi_{1}^{+} \phi_{2}^{-}\left(\lambda_{48} \varphi_{1}^{0} \varphi_{2}^{0 *}+\lambda_{49} \varphi_{1}^{0 *} \varphi_{2}^{0 *}+\lambda_{50} \varphi_{2}^{0} \xi^{0 *}\right) \\
& +\phi_{3}^{+} \phi_{4}^{-}\left(\lambda_{51} \varphi_{1}^{0} \xi^{0}+\lambda_{52} \varphi_{1}^{0} \xi^{0 *}+\lambda_{53} \varphi_{1}^{0 *} \xi^{0}+\lambda_{54} \varphi_{1}^{0 *} \xi^{0 *}\right) \\
& \left.+\left(\varphi_{2}^{0}\right)^{2}\left(\lambda_{55} \varphi_{1}^{0} \xi^{0}+\lambda_{56} \varphi_{1}^{0} \xi^{0 *}+\lambda_{57} \varphi_{1}^{0 *} \xi^{0}+\lambda_{58} \varphi_{1}^{0 *} \xi^{0 *}\right)+\mathrm{H.c.}\right\}
\end{aligned}
$$

The last part which breaks softly $Z_{4} \times Z_{2}$, is given by

$$
\begin{aligned}
L_{\mathrm{gsoft}}^{\mathrm{scalars}}= & \mu_{4}^{2} \varphi_{1}^{0} \varphi_{2}^{0}+\mu_{6}^{2} \varphi_{1}^{0} \varphi_{2}^{0 *}+\mu_{1}^{2}\left(\varphi_{2}^{0}\right)^{2}+\mu_{2}^{2} \phi_{2}^{+} \phi_{3}^{-} \\
& +\mu_{5}^{2} \phi_{2}^{+} \phi_{4}^{-}+\mu_{3}^{2} \phi_{3}^{+} \phi_{4}^{-}+\text {H.c. }
\end{aligned}
$$

The total potential is composed of three above mentioned parts

$$
V=V_{\mathrm{LNC}}+V_{\mathrm{LNV}}+\mathcal{L}_{\mathrm{soft}}^{\text {scalars }}
$$

The scalar interactions needed for quark and charged lepton mass generation, read as follows

$$
\begin{aligned}
L_{\text {Higgsqcl }}= & \lambda_{1} \chi \rho \eta \varphi_{1}^{0}+\lambda_{3} \eta^{\dagger} \rho \phi_{3}^{-} \xi^{0}+\lambda_{4} \phi_{1}^{+} \phi_{2}^{-} \varphi_{2}^{0} \xi^{0} \\
& +w_{1}\left(\varphi_{2}^{0}\right)^{2} \varphi_{1}^{0}+w_{2} \chi^{\dagger} \rho \phi_{3}^{-}+\text {H.c. }
\end{aligned}
$$

For the neutrino mass generation, beside the first term in (A5), the additional part is given as

$$
\begin{aligned}
L_{\text {Higgsneutrino }}= & \lambda_{13}\left(\chi^{\dagger} \chi\right)^{2}+\lambda_{5}\left(\chi^{\dagger} \chi\right)\left(\eta^{\dagger} \eta\right) \\
& +\left[\lambda_{27}\left(\rho^{\dagger} \rho\right)\left(\chi^{\dagger} \eta+\eta^{\dagger} \chi\right)+\mu_{3}^{2} \phi_{4}^{-} \phi_{3}^{+}+\text {H.c }\right] .
\end{aligned}
$$

It is worth mentioning that for the generation of masses for quark and charged lepton, only terms in the conserving part $V_{\text {LNC }}$ are enough, while for the generation of the light active neutrino masses, one needs the lepton number violating scalar interactions of $V_{\mathrm{LNV}}$ as well as the softly breaking part $\mathcal{L}_{\text {soft }}^{\text {scalars }}$ [the last term in (A6)] of the scalar potential. 
[1] C. D. Froggatt and H. B. Nielsen, Hierarchy of quark masses, Cabibbo angles and $C P$ violation, Nucl. Phys. B147, 277 (1979).

[2] A. E. Cárcamo Hernández, S. Kovalenko, and I. Schmidt, Radiatively generated hierarchy of lepton and quark masses, J. High Energy Phys. 02 (2017) 125.

[3] M. Singer, J. W. F. Valle, and J. Schechter, Canonical neutral current predictions from the weak electromagnetic gauge group $S U(3) \times U(1)$, Phys. Rev. D 22, 738 (1980).

[4] J. W. F. Valle and M. Singer, Lepton number violation with quasi dirac neutrinos, Phys. Rev. D 28, 540 (1983).

[5] F. Pisano and V. Pleitez, An $S U(3) \times U(1)$ model for electroweak interactions, Phys. Rev. D 46, 410 (1992).

[6] R. Foot, O. F. Hernandez, F. Pisano, and V. Pleitez, Lepton masses in an $S U(3)_{L} \times U(1)_{N}$ gauge model, Phys. Rev. D 47, 4158 (1993).

[7] P. H. Frampton, Chiral Dilepton Model and the Flavor Question, Phys. Rev. Lett. 69, 2889 (1992).

[8] H. N. Long, $S U(3)_{L} \times U(1)_{N}$ model for right-handed neutrino neutral currents, Phys. Rev. D 54, 4691 (1996).

[9] H. N. Long, The 331 model with right handed neutrinos, Phys. Rev. D 53, 437 (1996).

[10] R. Foot, H. N. Long, and T. A. Tran, $S U(3)_{L} \otimes U(1)_{N}$ and $S U(4)_{L} \otimes U(1)_{N}$ gauge models with right-handed neutrinos, Phys. Rev. D 50, R34 (1994).

[11] C. A. de Sousa Pires and O. P. Ravinez, Charge quantization in a chiral bilepton gauge model, Phys. Rev. D 58, 035008 (1998).

[12] P. V. Dong and H. N. Long, Electric charge quantization in $S U(3)_{C} \times S U(3)_{L} \times U(1)_{X}$ models, Int. J. Mod. Phys. A 21, 6677 (2006).

[13] J. C. Montero, V. Pleitez, and O. Ravinez, Soft superweak CP violation in a 331 model, Phys. Rev. D 60, 076003 (1999).

[14] J. C. Montero, C. C. Nishi, V. Pleitez, O. Ravinez, and M. C. Rodriguez, Soft $C P$ violation in $K$ meson systems, Phys. Rev. D 73, 016003 (2006).

[15] P. B. Pal, The strong $C P$ question in $S U(3)_{C} \times S U(3)_{L} \times$ $U(1)_{N}$ models, Phys. Rev. D 52, 1659 (1995).

[16] A. G. Dias, V. Pleitez, and M. D. Tonasse, Naturally light invisible axion in models with large local discrete symmetries, Phys. Rev. D 67, 095008 (2003).

[17] A. G. Dias and V. Pleitez, Stabilizing the invisible axion in 3-3-1 models, Phys. Rev. D 69, 077702 (2004).

[18] A. G. Dias, C. A. de S. Pires, and P. S. Rodrigues da Silva, Discrete symmetries, invisible axion and lepton number symmetry in an economic 3-3-1 model, Phys. Rev. D 68, 115009 (2003).

[19] A. E. Cárcamo Hernández, S. Kovalenko, H. N. Long, and I. Schmidt, A variant of 3-3-1 model for the generation of the SM fermion mass and mixing pattern, J. High Energy Phys. 07 (2018) 144.

[20] K. Huitu and N. Koivunen, Froggatt-Nielsen mechanism in a model with $S U(3)_{c} \times S U(3)_{L} \times U(1)_{X}$ gauge group, Phys. Rev. D 98, 011701 (2018).

[21] D. Chang and H. N. Long, Interesting radiative patterns of neutrino mass in an $S U(3)_{c} \times S U(3)_{L} \times U(1)_{X}$ model with right-handed neutrinos, Phys. Rev. D 73, 053006 (2006).

[22] C. Salazar, R. H. Benavides, W. A. Ponce, and E. Rojas, LHC constraints on 3-3-1 models, J. High Energy Phys. 07 (2015) 096.
[23] A. J. Buras, F. De Fazio, J. Girrbach, and M. V. Carlucci, The anatomy of quark flavour observables in 331 models in the flavour precision era, J. High Energy Phys. 02 (2013) 023.

[24] A. E. Cárcamo Hernández, R. Martinez, and F. Ochoa, Z and Z' decays with and without FCNC in 331 models, Phys. Rev. D 73, 035007 (2006).

[25] R. Martinez and F. Ochoa, Mass-matrix ansatz and constraints on B0(s) - anti-B0(s) mixing in 331 models, Phys. Rev. D 77, 065012 (2008).

[26] A. J. Buras, F. De Fazio, and J. Girrbach, 331 models facing new $b \rightarrow s \mu^{+} \mu^{-}$data, J. High Energy Phys. 02 (2014) 112.

[27] A. J. Buras, F. De Fazio, and J. Girrbach-Noe, $Z-Z^{\prime}$ mixing and $Z$-mediated FCNCs in $S U(3)_{C} \times S U(3)_{L} \times$ $U(1)_{X}$ models, J. High Energy Phys. 08 (2014) 039.

[28] V. T. N. Huyen, H. N. Long, T. T. Lam, and V. Q. Phong, Neutral current in reduced minimal 3-3-1 model, Commun. Phys. 24, 97 (2014).

[29] H. N. Long and T. Inami, S, T, U parameters in $S U(3)_{C} \times$ $S U(3)_{L} \times U(1)_{X}$ model with right-handed neutrinos, Phys. Rev. D 61, 075002 (2000).

[30] M. Tanabashi et al. (Particle Data Group), Review of particle physics, Phys. Rev. D 98, 030001 (2018).

[31] P. V. Dong, H. N. Long, D. T. Nhung, and D. V. Soa, $S U(3)_{C} \times S U(3)_{L} \times U(1)_{X}$ model with two Higgs triplets, Phys. Rev. D 73, 035004 (2006).

[32] F. S. Queiroz, C. Siqueira, and J. W. F. Valle, Constraining flavor changing interactions from LHC run-2 dilepton bounds with vector mediators, Phys. Lett. B 763, 269 (2016).

[33] V. Q. Phong, N. T. Tuong, N. C. Thao, and H. N. Long, Multiperiod structure of electroweak phase transition in the 3-3-1-1 model, Phys. Rev. D 99, 015035 (2019).

[34] M. J. Baker, M. Breitbach, J. Kopp, and L. Mittnacht, Dynamic freeze-in: Impact of thermal masses and cosmological phase transitions on dark matter production, J. High Energy Phys. 03 (2018) 114.

[35] D. López-Val and T. Robens, $\Delta r$ and the W-boson mass in the singlet extension of the standard model, Phys. Rev. D 90, 114018 (2014).

[36] A. E. Cárcamo Hernández, I. de Medeiros Varzielas, and E. Schumacher, Fermion and scalar phenomenology of a twoHiggs-doublet model with $S_{3}$, Phys. Rev. D 93, 016003 (2016).

[37] D. Lopez-Val and J. Sola, Delta $r$ in the two-Higgs-doublet model at full one loop level—and beyond, Eur. Phys. J. C 73, 2393 (2013).

[38] M. Drees and K. Hagiwara, Supersymmetric contribution to the electroweak $\rho$ parameter, Phys. Rev. D 42, 1709 (1990).

[39] M. Aaboud et al. (ATLAS Collaboration), Search for additional heavy neutral Higgs and gauge bosons in the ditau final state produced in $36 \mathrm{fb}^{-1}$ of pp collisions at $\sqrt{s}=13 \mathrm{TeV}$ with the ATLAS detector, J. High Energy Phys. 01 (2018) 055.

[40] M. Aaboud et al. (ATLAS Collaboration), Search for charged Higgs bosons decaying via $H^{ \pm} \rightarrow \tau^{ \pm} \nu_{\tau}$ in the $\tau+$ jets and $\tau+$ lepton final states with $36 \mathrm{fb}^{-1}$ of $p p$ collision data recorded at $\sqrt{s}=13 \mathrm{TeV}$ with the ATLAS experiment, J. High Energy Phys. 09 (2018) 139. 
[41] M. Aaboud et al. (ATLAS Collaboration), Search for heavy resonances decaying to a photon and a hadronically decaying $Z / W / H$ boson in $p p$ collisions at $\sqrt{s}=13 \mathrm{TeV}$ with the ATLAS detector, Phys. Rev. D 98, 032015 (2018).

[42] V. Khachatryan et al. (CMS Collaboration), Search for high-mass diphoton resonances in protonproton collisions at $13 \mathrm{TeV}$ and combination with $8 \mathrm{TeV}$ search, Phys. Lett. B 767, 147 (2017).

[43] M. Aaboud et al. (ATLAS Collaboration), Search for new phenomena in high-mass diphoton final states using $37 \mathrm{fb}^{-1}$ of proton-proton collisions collected at $\sqrt{s}=13 \mathrm{TeV}$ with the ATLAS detector, Phys. Lett. B 775, 105 (2017).

[44] F. Deppisch and J. W. F. Valle, Enhanced lepton flavor violation in the supersymmetric inverse seesaw model, Phys. Rev. D 72, 036001 (2005).

[45] A. Ilakovac and A. Pilaftsis, Flavour-violating charged lepton decays in seesaw-type models, Nucl. Phys. B437, 491 (1995).

[46] M. Lindner, M. Platscher, and F. S. Queiroz, A call for new physics: The muon anomalous magnetic moment and lepton flavor violation, Phys. Rep. 731, 1 (2018).
[47] M. A. Perez, G. Tavares-Velasco, and J. J. Toscano, Twobody $Z^{\prime}$ decays in the minimal 3-3-1 model, Phys. Rev. D 69, 115004 (2004).

[48] G. C. Branco, P. M. Ferreira, L. Lavoura, M. N. Rebelo, M. Sher, and J. P. Silva, Theory and phenomenology of twoHiggs-doublet models, Phys. Rep. 516, 1 (2012).

[49] J. Edsjo and P. Gondolo, Neutralino relic density including coannihilations, Phys. Rev. D 56, 1879 (1997).

[50] S. Bhattacharya, P. Poulose, and P. Ghosh, Multipartite interacting scalar dark matter in the light of updated LUX data, J. Cosmol. Astropart. Phys. 04 (2017) 043.

[51] J. Elias-Miro, J. R. Espinosa, G. F. Giudice, H. M. Lee, and A. Strumia, Stabilization of the electroweak vacuum by a scalar threshold effect, J. High Energy Phys. 06 (2012) 031.

[52] K. Kannike, Vacuum stability of a general scalar potential of a few fields, Eur. Phys. J. C 76, 324 (2016).

[53] G. Cynolter, E. Lendvai, and G. Pocsik, Note on unitarity constraints in a model for a singlet scalar dark matter candidate, Acta Phys. Pol. B 36, 827 (2005).

[54] P. A. R. Ade et al. (Planck Collaboration), Planck 2015 results. XIII. Cosmological parameters, Astron. Astrophys. 594, A13 (2016). 